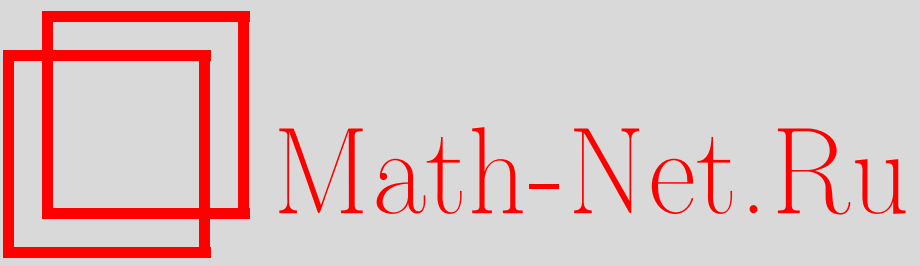

А. Ю. Борисович, Бифуркация капиллярной минимальной поверхности в слабом гравитационном поле, Матем. сб., 1997, том 188, номер 3, 17-48

DOI: https://doi.org/10.4213/sm209

Использование Общероссийского математического портала Math-Net.Ru подразумевает, что вы прочитали и согласны с пользовательским соглашением

http://www.mathnet.ru/rus/agreement

Параметры загрузки:

IP : 54.205 .225 .156

26 апреля 2023 г., 15:05:32 
УДК 517

\author{
А. Ю. Борисович
}

\title{
Бифуркация капиллярной минимальной поверхности в слабом гравитационном поле
}

\begin{abstract}
В работе исследуется одна вариационная краевая эллиптическая задача в выпуклой области $\Omega \subset \mathbb{R}^{2}$ с параметром Бонда $\lambda \in \mathbb{R}$, возникшая в гидромеханике и тесно связанная с проблемой Плато. Она описывает поведение эластичной поверхности, разделяющей две жидкие или газообразные среды, при изменении гравитационного поля. При отсутствии силы тяжести $\lambda=0$ и решением задачи является минимальная поверхность. В работе исследуется поведение этой поверхности при появлении гравитации (потеря устойчивости, бифуркации).

Метод исследования основан на редукции задачи к операторному уравнению в пространствах Гёльдера или Соболева с нелинейным фредгольмовым индекса 0 оператором, зависящим от параметра $\lambda$, и на применении к полученному уравнению теоремы Крендала-Рабиновича о простой точке бифуркации, метода конечномерной редукции Ляпунова-Шмидта, метода ключевой функции Ю.И. Сапронова. В работе получены общие как необходимые, так и достаточные условия бифуркации в данной задаче. Детально исследована ситуация, когда область $\Omega$ круг или квадрат.

Библиографоя: 27 названий.
\end{abstract}

\section{0. Введение}

Уравнение поверхности, разделяющей две жидкие среды, впервые появилось в работах Юнга, Пуассона, Лапласа и Монжа. Они заметили, что средняя кривизна поверхности раздела в каждой ее точке постоянна и пропорциональна разности давлений на эту поверхность с двух сторон. Если две жидкости находятся в капилляре и граница разделяющей их поверхности свободна, т.е. не закреплена в определенном положении, то она соединяется с боковой поверхностью капилляра под определенным углом $\gamma$, зависяшим от коэффициентов смачивания стенки капилляра этими жидкостями.

Пусть $\Omega$ - перпендикулярное сечение капилляра и поверхность раздела задана как график функции $w=w(x, y),(x, y) \in \bar{\Omega}$. Тогда, ее средняя кривизна $H(w)(x, y)$ вычисляется по формуле:

$$
H(w)=-\operatorname{div} T(w), \quad T(w)=\left(1+\nabla w^{2}\right)^{-1 / 2} \nabla w .
$$

Работа выполнена при поддержке Российского фонда фундаментальных исследований (грант № 94-01-00413), Международного научного фонда и UG (грант BW 5100-5-0053-6). 
Пусть $\bar{n}$ - вектор нормали к поверхности, а $\bar{\eta}$ - вектор внутренней нормали к боковой поверхности капилляра

$$
\begin{array}{ll}
\bar{n}=\left(1+\nabla w^{2}\right)^{-1 / 2}\left(w_{x}, w_{y},-1\right), & |\bar{n}|=1, \\
\bar{\eta}=\left(\nu_{x}, \nu_{y}, 0\right), & |\bar{\eta}|=1 .
\end{array}
$$

Тогда $\overline{\eta n}=\nu T(w)$, где $\nu=\left(\nu_{x}, \nu_{y}\right)$, и капиллярная поверхность $w(x, y)$ определяется следующей краевой задачей

$$
\begin{cases}-\operatorname{div} T(w)=\varkappa \Delta p, & (x, y) \in \Omega, \\ \nu T(w)=\cos \gamma, & (x, y) \in \partial \Omega,\end{cases}
$$

где $\Delta p=p_{2}-p_{1}-$ разность давлений на поверхность с двух сторон, $\varkappa=1 / \sigma$, и $\sigma>0-$ коэффищиент поверхностного натяжения.

Если две жидкости находятся в капилляре и граница разделяющей их поверхности закреплена в определенном положении, то для самой поверхности получаем задачу типа Дирихле с краевым условием $\left.w\right|_{\partial \Omega}=\psi, \psi: \partial \Omega \rightarrow \mathbb{R}$.

В некоторых ситуациях разность давлений $\Delta p$ равна нулю. Например, в условиях невесомости или в случае, когда поверхность $w(x, y)$ разделяет две несмешиваемые жидкости одинаковой плотности. В этом случае средняя кривизна разделяющей поверхности $H(w) \equiv 0$. Такие поверхности называются минимальньми, так как одновременно они являются критическими точками функционала площади, определенного на пространстве поверхностей.

Гаусс предложил вариационный подход к описанию капиллярных поверхностей. В задаче со свободной границей (0.2) они ищутся как критические точки функционала потенциальной энергии

$$
E_{\text {complet }}(w)=E_{\sigma}(w)+E_{g}(w)+E_{2}(\partial w)+E_{1}(\partial w)
$$

где

$$
E_{\sigma}(w)=\sigma \iint_{\Omega}\left(1+\nabla w^{2}\right)^{1 / 2} d x d y
$$

- потенциальная энергия поверхностного натяжения, которая прямо пропорциональна площади поверхности ( $\sigma$ - коэффициент поверхностного натяжения), $E_{g}(w)$ - потенциальная энергия жидкости в гравитационном поле ( $g$ - ускорение свободного падения), $E_{2}(\partial w)$ и $E_{1}(\partial w)$ - энергии взаимодействия жидкостей со стенками капилляра, которые зависят только от положения границы поверхности $w(x, y)$ и от коэффициентов смачивания стенки капилляра этими жидкостями. В задаче с фиксированной границей последние два слагаемых в формуле (0.3) являются постоянными и не влияют на форму поверхности раздела. В этом случае гидромеханические модели отличаются друг от друга слагаемым $E_{g}(w)$.

Проблема сушествования решения задачи (0.2) для различных гидромеханических моделей исследовалась Релеем, Нейманом, Минковским, Пуанкаре и многими другими авторами, в том числе современными. Обзор этих исследований можно найти в фундаментальных монографиях А. T. Фоменко [1], [2], V. Dissan, S. Davis [3], [4], R. Finn [5]. В последнее время в связи с развитием космической техники 
возрастает интерес к гидромеханике невесомости (см., например, работы [6], [7]). В статьях [8], [9] исследуется движение в условиях невесомости твердого тела с полостью, частично заполненной вязкой жидкостью.

С другой стороны, в математике с 1972 года активно исследуется проблема бифуркации минимальных поверхностей при малых возмущениях граничных условий и функционала плошади: Т. Poston [10], M. J. Beeson, A. J. Tromba [11], А. Т. Фоменко, А. А. Тужилин [12], [13], J. Buch [14], А. Ю. Борисович [15]. В работе [10] содержится описание физических экспериментов в которых было обнаружено и исследовано явление бифуркации минимальных поверхностей. Она послужила толчком для математического исследования этого явления. В работах [11], [14] для исследования бифуркаций минимальных поверхностей используются методы нелинейного функционального анализа. В работе [12], например, исследуются ветвления графика многозначного функционала площади минимальных поверхностей, определенного на пространстве контуров. В работах [12], [13] развиваются геометрические методы. В [15] автором построен обший функционально-операторный метод исследования бифуркаций параметрически заданных минимальных поверхностей при достаточно обших малых деформациях граничного контура. Метод оказался эффективньм в приложениях. Он позволил обнаружить и исследовать целые серии новых бифуркаций на классических минимальных поверхностях: катеноиде, геликоиде, поверхностях Шерка и Эннепера.

В данной работе рассматривается непараметрический случай и исследуются бифуркации минимальной поверхности раздела двух сред при фиксированной границе и квадратичном возмушении функционала плошади. Изучаемая модель возникает в различных задачах гидромеханики и теории упругих мембран. Например, при движении в условиях невесомости твердого тела (ракеты) с полостью, заполненной вязкой жидкостью (топливом) и имеюшей капиллярное отверстие.

\section{1. Постановка задачи и ее обсуждение}

В данной работе исследуются бифуркации семейств решений следуюшей вариационной краевой задачи

$$
\begin{gathered}
E(w, \lambda)=\iint_{\Omega}\left(\left(1+\nabla w^{2}\right)^{1 / 2}-\frac{\lambda}{2}\left(w-w_{0}\right)^{2}\right) d x d y, \\
\begin{cases}-\operatorname{div} T(w)-\lambda\left(w-w_{0}\right)=0, & (x, y) \in \Omega, \\
w=\psi(\mu), & (x, y) \in \partial \Omega,\end{cases}
\end{gathered}
$$

определенной в области $\Omega \in \mathbb{R}^{2}$, зависяшей от основного параметра $\lambda \in \mathbb{R}$ и дополнительного параметра $\mu \in \mathbb{R}_{\varepsilon}=(-\varepsilon,+\varepsilon)$, которьй задает малое возмущение граничных условий.

Здесь нижними индексами $x$ и $y$ обозначены частные производные функции $w(x, y)$ по переменным $x$ и $y$, соответственно,

$$
\begin{gathered}
\nabla w^{2}=\left(w_{x}\right)^{2}+\left(w_{y}\right)^{2}, \quad T(w)=\left(1+\nabla w^{2}\right)^{-1 / 2} \nabla w, \quad \Delta w=w_{x x}+w_{y y} \\
-\operatorname{div} T(w)=-\left(1+\nabla w^{2}\right)^{-3 / 2}\left(\Delta w+w_{y}^{2} w_{x x}-2 w_{x} w_{y} w_{x y}+w_{x}^{2} w_{y y}\right)
\end{gathered}
$$


Задача (1.1)-(1.2) исследуется при следующих предположениях.

ПРЕДПОЛОЖЕНИЕ $\mathrm{A}_{1} . w_{0}(x, y)$ является минимальной поверхностью с граничей $\psi_{0}: \partial \Omega \rightarrow \mathbb{R}$

$$
\left\{\begin{array}{l}
\Delta w_{0}+w_{0 y}^{2} w_{0 x x}-2 w_{0 x} w_{0 y} w_{0 x y}+w_{0 x}^{2} w_{0 y y}=0 \\
\left.w_{0}\right|_{\partial \Omega}=\psi_{0}
\end{array}\right.
$$

ПРЕДПОЛОЖЕНИЕ $\mathrm{A}_{2}$. Область $\Omega$ является выпуклой, а ее граница $\partial \Omega$ является кусочно-гладким $C^{2}$-подмногообразием в $\mathbb{R}^{2}$, гомеоморфным стандартной окружсности $S^{1}$.

ПРЕДПОЛОЖЕнИЕ $\mathrm{A}_{3}$. Семейство отображений $\psi: \partial \Omega \times \mathbb{R}_{\varepsilon} \rightarrow \mathbb{R}$ является достаточно гладким и удовлетворяет начальному условию

$$
\psi(0)=\psi_{0} .
$$

Предположение $\mathrm{A}_{1}$ обеспечивает сушествование при $\mu=0$ одного семейства решений

$$
\Gamma_{1}=\left\{\left(w_{0}, \lambda\right): \lambda \in \mathbb{R}\right\},
$$

и нас будет интересовать наличие других ветвей решений, пересекающих $\Gamma_{1}$.

Изучаемьй функционал $E(w, \lambda)$ может быть записан в виде

$$
E(w, \lambda)=A(w)-\lambda Q(w),
$$

и является квадратичной однопараметрической деформацией функционала плошади поверхности

$$
A(w)=\iint_{\Omega}\left(1+\nabla w^{2}\right)^{1 / 2} d x d y, \quad Q(w)=\frac{1}{2} \iint_{\Omega}\left(w-w_{0}\right)^{2} d x d y .
$$

Эллиптический дифференшиальный оператор

$$
F(w, \lambda)=-\operatorname{div} T(w)-\lambda\left(w-w_{0}\right)
$$

является оператором Эйлера-Лагранжа для функционала $E(w, \lambda)$.

Действительно, рассмотрим малую вариацию поверхности $h=\delta w$ при условии $\left.h\right|_{\partial \Omega}=0$ и выгислим первую вариацию функционала $E$

$$
\begin{aligned}
\delta E & =\iint_{\Omega}\left(\left(1+\nabla w^{2}\right)^{1 / 2} \nabla w \nabla h-\lambda\left(w-w_{0}\right) h\right) d x d y \\
& =\iint_{\Omega}\left(-\operatorname{div}\left(\left(1+\nabla w^{2}\right)^{-1 / 2} \nabla w\right)-\lambda\left(w-w_{0}\right)\right) h d x d y .
\end{aligned}
$$

Тогда критические точки (поверхности) функционала $E$ определяются краевой задачей (1.2) с параметром $\lambda \in \mathbb{R}$. 
Задача (1.2) при $\lambda=\mu=0$ является непараметрической проблемой Плато и исследовалась многими авторами. Например, первые теоремы существования и единственности были доказаны С. Н. Бернштейном [16]. В работе [17] установлено, что оператор

$$
G(w)=\left(\Delta w+w_{y}^{2} w_{x x}-2 w_{x} w_{y} w_{x y}+w_{x}^{2} w_{y y},\left.w\right|_{\partial \Omega}\right)
$$

является глобальным $C^{1}$-диффеоморфизмом пространств Гёльдера

$$
G: C^{m, \alpha}(\bar{\Omega}) \rightarrow C^{m-2, \alpha}(\bar{\Omega}) \times C^{m, \alpha}(\partial \Omega)
$$

с целым $m \geqslant 2$ и $0<\alpha<1$, если область $\Omega$ строго выпукла и ее граница $\partial \Omega$ является гладким подмногообразием класса $C^{m, \alpha}$.

Пусть $\mu=0$. Тогда, при каждом $\lambda \in \mathbb{R}$ минимальная поверхность $w_{0}(x, y)$ будет решением задачи (1.2). При отрищательных и малых положительных $\lambda$ это решение будет единственным и, более того, устойчивым.

Действительно, вычислим вторую вариацию функционала $E$

$$
\begin{aligned}
\delta^{2} E & =\delta^{2} \iint_{\Omega}\left(1+\nabla w^{2}\right)^{1 / 2} d x d y-\lambda \delta^{2} \iint_{\Omega} 1 / 2\left(w-w_{0}\right)^{2} d x d y \\
& =\iint_{\Omega}\left(\left(1+\nabla w^{2}\right)^{-3 / 2}\left\{\nabla h^{2}+\nabla w^{2} \nabla h^{2}-(\nabla w \nabla h)^{2}\right\}-\lambda h^{2}\right) d x d y .
\end{aligned}
$$

При $\lambda \leqslant 0$ вторая вариация является положительно определенной квадратичной формой $\left(\nabla w^{2} \nabla h^{2} \geqslant(\nabla w \nabla h)^{2}\right)$ и, следовательно, $w_{0}$ будет точкой минимума функционала $E$. Подобная ситуация сохранится при малых положительных $\lambda$. При росте $\lambda$ будет увеличиваться влияние второго функционала в формуле (1.8), для которого $w_{0}$ - точка максимума. В результате, при некотором положительном значении параметра $\lambda$ произойдет потеря устойчивости решения $w_{0}$ задачи (1.1)-(1.2). В данной работе показано, что потеря устойчивости связана с ответвлением от $w_{0}$ других семейств устойчивых решений.

Семейство решений $\Gamma_{1}$ образовано минимальными поверхностями, которые, как известно, являются аналитическими в каждой внутренней точке. Поверхности, образующие другие ветви решений, уже не будут минимальными и вопрос о их регулярности исследуется в данной работе в зависимости от класса гладкости границы $\partial \Omega$, количества и характера угловых точек на ней.

Рассмотрим гидромеханическую проблему, соответствуюшую задаче (1.1)-(1.2).

В цилиндре с поперечным сечением $\Omega$ находятся две жидкие или газообразные среды с плотностью $\rho_{1}$ и $\rho_{2}$ соответственно (рис. 1 ), $\rho=\rho_{2}-\rho_{1}>0$. Разделяющая их эластичная поверхность

$$
w=w(x, y), \quad(x, y) \in \bar{\Omega}
$$

зафиксирована на боковой поверхности цилиндра

$$
\left.w\right|_{\partial \Omega}=\psi_{0}
$$




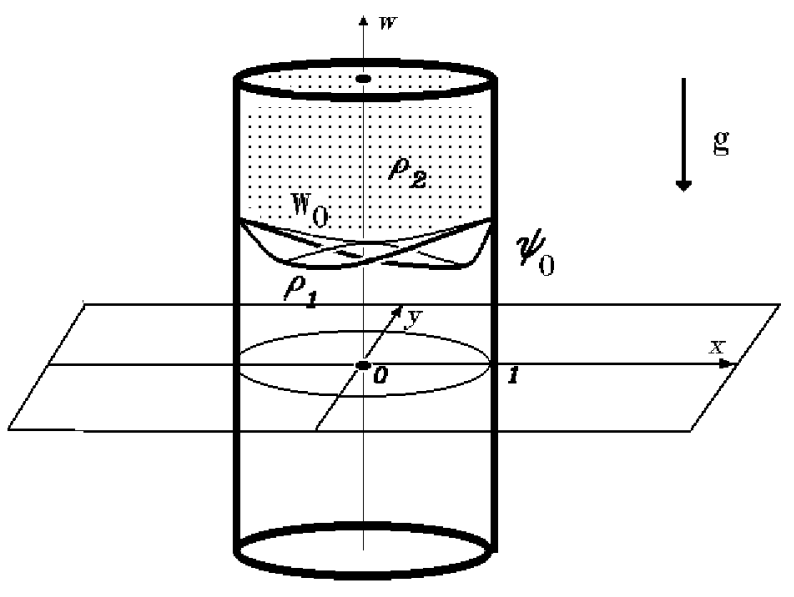

Рис. 1

с помошью непрерывного отображения $\psi_{0}: \partial \Omega \rightarrow \mathbb{R}$. Пусть $\sigma>0$ - коэффищиент поверхностного натяжения.

При отсутствии гравитации поверхность раздела $w_{0}(x, y)$ будет точкой абсолютного минимума функционала поверхностной энергии $E_{\sigma}(w)=\sigma A(w)$ и, следовательно, минимальной поверхностью.

При появлении ускорения $g>0$ к потенциальной энергии поверхностного натяжения $E_{\sigma}(w)$ добавится потенциальная энергия двух сред в цилиндре $E_{g}(w)$. Рассматриваемый цилиндр может быть частью различных гидромеханических систем и это определяет соответствуюшую формулу для $E_{g}$. В данной работе исследуется ситуация, когда потенциальная энергия выгисляется по формуле

$$
E_{g}(w)=E_{0}-\rho g Q(w), \quad Q(w)=\frac{1}{2} \iint_{\Omega}\left(w-w_{0}\right)^{2} d x d y
$$

Тогда поверхность раздела двух сред $w(x, y)$ будет критической точкой суммарного функционала энергии

$$
E_{\sigma}+E_{g}=E_{0}+\sigma A(w)-\rho g Q(w)=E_{0}+\sigma(A(w)-\lambda Q(w)),
$$

зависяшего от параметра Бонда $\lambda=\rho g / \sigma$, т.е. решением задачи (1.1)-(1.2).

Рассмотрим, в каких гидромеханических моделях возникает функционал (1.15). Пусть цилиндр, изображенный на рис. 1, является капилляром и содержит определенньй объем жидкости, удерживаемьй капиллярной поверхностью $w$. Тогда, потенциальная энергия столба жидкости определяется положением его нижней и верхней границ и вычисляется по формуле

$$
E_{g}(w)=E_{0}-\rho g \iint_{\Omega} w^{2} d x d y+L \iint_{\Omega} w d x d y
$$

введенной Гауссом и Лежандром, где $L$ - константа Лежандра. Если капилляр соединен с большим объемом жидкости, то учитьвают изменение только нижней 
границы и используют функционал (1.17) без последнего слагаемого. Теоретически, это соответствует случаю бесконечного цилиндра. Если жидкость является достаточно вязкой и отклонение $w(x, y)-w_{0}(x, y)$ мало, то невозможно перемещение объемов жидкости вдоль поверхности $w_{0}$ на значительные расстояния. В последней ситуации используют функционал в форме (1.15).

Пусть, например, в условиях невесомости двигается твердое тело с полостью, заполненной вязкой жидкостью, и в нем имеется капиллярное отверстие. Тогда, за счет сил поверхностного натяжения образуется капиллярная поверхность $w_{0}(x, y)$ (минимальная), прикрепленная к краю отверстия, и препятствующая вытеканию жидкости. При возникновении ускорения $g$ форма этой поверхности будет определяться функционалом (1.15).

\section{2. Редукция задачи к операторному уравнению с нелинейным фредгольмовым индекса 0 оператором}

Минимальная поверхность $w_{0}(x, y)$ будет решением задачи $(1.1)-(1.2)$ при всех $\lambda \in \mathbb{R}$ и проблема ее бифуркаций эквивалентна задаче о ветвлении семейств решений операторного уравнения

$$
P(w, \lambda, \mu) \equiv\left(F(w, \lambda),\left.w\right|_{\partial \Omega}-\psi(\mu)\right)=(0,0)
$$

в точках $\left\{\left(w_{0}, \lambda, 0\right): \lambda \in \mathbb{R}\right\}$, где $P\left(w_{0}, \lambda, 0\right)=(0,0)$.

Исследуем задачу (1.1)-(1.2) и операторное уравнение (2.1) в случае гладкой границы $\partial \Omega$. Это позволяет искать ответвляюшиеся от $w_{0}$ решения в классе функций такой же гладкости, что и сама гранища.

Исследуем задачу (1.1)-(1.2) при следующих дополнительных предположениях.

ПРЕДПОЛОЖЕНИЕ $\mathrm{A}_{2}^{\prime}$. Область $\Omega$ (поперечное сечение капилляра) строго выпукла и ее граница $\partial \Omega$ является гладким подмногообразием класса $C^{m, \alpha}$ с иельм $m \geqslant 2 u 0<\alpha<1$.

ПРЕДПОЛОЖЕНИЕ $\mathrm{A}_{3}^{\prime}$. Отображение $\psi_{0}$ принадлежит классу $C^{m, \alpha}(\partial \Omega)$. Семейство отображсений $\psi: C^{m, \alpha}(\partial \Omega) \times \mathbb{R}_{\varepsilon} \rightarrow \mathbb{R}$ является аналитическим по совокупности переменных и удовлетворяет начальному условию $\psi(0)=\psi_{0}$.

Теорема 2.1. При предположениях $\mathrm{A}_{1}, \mathrm{~A}_{2}^{\prime}, \mathrm{A}_{3}^{\prime}$ нелинейный оператор $P$ действует в пространствах Гёльдера

$$
P: C^{m, \alpha}(\bar{\Omega}) \times \mathbb{R} \times \mathbb{R}_{\varepsilon} \rightarrow C^{m-2, \alpha}(\bar{\Omega}) \times C^{m, \alpha}(\partial \Omega),
$$

является аналитическим по совокупности переменных и фредгольмовым индекса 0 по основной переменной $w$, т.е. класса $C^{a} \Phi_{0}^{(w)}$.

Доказательство. Действие оператора $P$ в пространствах $(2.2)$ и его гладкость устанавливаются как и в работе [17]. Вычислим, например, производную Фреше по $w$. Для этого вторую вариацию (1.13) функционала $E$ запишем в дивергентной форме

$$
\delta^{2} E=\sigma \iint_{\Omega}(A(w) h-\lambda h) h d x d y
$$


где $A(w)$ - производная Фреше от оператора средней кривизны $H(w)$ и

$$
\begin{aligned}
A(w) h= & -\operatorname{div}\left(\left(1+\nabla w^{2}\right)^{-3 / 2}\left[\begin{array}{cc}
1+w_{y}^{2} & w_{x} w_{y} \\
w_{x} w_{y} & 1+w_{x}^{2}
\end{array}\right] \times\left[\begin{array}{l}
h_{x} \\
h_{y}
\end{array}\right]\right) \\
= & -\left(1+\nabla w^{2}\right)^{-3 / 2}\left(\left(1+w_{y}^{2}\right) h_{x x}-2 w_{x} w_{y} h_{x y}+\left(1+w_{x}^{2}\right) h_{y y}\right. \\
& \left.+2\left(w_{x} w_{y y}-w_{y} w_{x y}\right) h_{x}+2\left(w_{y} w_{x x}-w_{x} w_{x y}\right) h_{y}\right) .
\end{aligned}
$$

Тогда дифференциал Фреше оператора $P$ будет определен по формуле

$$
\begin{gathered}
P_{w}^{\prime}(w, \lambda, \mu) h=\left(A(w) h-\lambda h,\left.h\right|_{\partial \Omega}\right), \\
P_{w}^{\prime}(w, \lambda): C^{m, \alpha}(\bar{\Omega}) \rightarrow C^{m-2, \alpha}(\bar{\Omega}) \times C^{m, \alpha}(\partial \Omega) .
\end{gathered}
$$

Он не зависит от $\mu$ и может быть представлен в виде

$$
P_{w}^{\prime}(w, \lambda) h=\left(A(w) h,\left.h\right|_{\partial \Omega}\right)-(\lambda h, 0) .
$$

Линейньй по $h$ дифференциальный оператор $A(w) h=-\operatorname{div}(\ldots)$ при каждом $w \in C^{m, \alpha}(\bar{\Omega})$ является равномерно эллиптическим

$$
\begin{gathered}
\omega\left(\xi_{1}, \xi_{2}\right)=\left(1+\nabla w^{2}\right)^{-3 / 2}\left(\left(1+w_{y}^{2}\right) \xi_{1}^{2}-2 w_{x} w_{y} \xi_{1} \xi_{2}+\left(1+w_{x}^{2}\right) \xi_{2}^{2}\right) \\
=\left(1+\nabla w^{2}\right)^{-3 / 2}\left(\xi_{1}^{2}+\left(w_{y} \xi_{1}-w_{x} \xi_{2}\right)^{2}+\xi_{2}^{2}\right), \\
\left(1+\nabla w^{2}\right)^{-3 / 2}\left(\xi_{1}^{2}+\xi_{2}^{2}\right) \leqslant \omega\left(\xi_{1}, \xi_{2}\right) \leqslant\left(1+\nabla w^{2}\right)^{-3 / 2}\left(\left(1+2 w_{y}^{2}\right) \xi_{1}^{2}+\left(1+2 w_{x}^{2}\right) \xi_{2}^{2}\right), \\
\left(1+2 C^{2}\right)^{-3 / 2}\left(\xi_{1}^{2}+\xi_{2}^{2}\right) \leqslant \omega\left(\xi_{1}, \xi_{2}\right) \leqslant\left(1+2 C^{2}\right)\left(\xi_{1}^{2}+\xi_{2}^{2}\right),
\end{gathered}
$$

где $C=\max _{(x, y) \in \bar{\Omega}}\left\{\left|w_{x}\right|,\left|w_{y}\right|\right\}$. Коэффициенты оператора принадлежат классу $C^{m-2, \alpha}(\bar{\Omega})$.

Первый оператор в формуле (2.6) будет линейным изоморфизмом пространств Гёльдера (см., например, [18]) и, следовательно, линейным фредгольмовым оператором индекса 0 . Второй оператор в формуле (2.6) будет вполне непрерывным в силу вполне непрерьвного вложения пространств Гёльдера и его добавление не влияет на фредгольмовость и индекс.

Следовательно, производная $\Phi$ реше $P_{w}^{\prime}(w, \lambda, \mu)$ в каждой точке $(w, \lambda, \mu)$ является линейньм оператором класса $\Phi_{0}$, а $P$ является нелинейным оператором класса $C^{a} \Phi_{0}^{(w)}$.

Исследуем задачу (1.1)-(1.2) и операторное уравнение (2.1) в случае кусочно-гладкой границы $\partial \Omega$. Для области с углами будем использовать при $p>2$ пространства $L_{p}(\Omega)$, пространства Соболева $W_{p}^{2}(\Omega)$ и пространства Бесова $B_{p}^{2-1 / p}(\partial \Omega)$, которые являются пространствами следов для $W_{p}^{2}(\Omega)$.

Исследуем задачу (1.1)-(1.2) при следующих дополнительных предположениях.

ПРЕДПОЛОЖЕНИЕ $\mathrm{A}_{2}^{\prime \prime}$. Область $\Omega$ (поперечное сечение капилляра) выпукла. Ее граница $\partial \Omega$ является кусочно-гладким $C^{2}$-подмногообразием в $\mathbb{R}^{2}$, гомеоморфным окружности $S^{1}$ и имеющим конечное число $K$ угловых точек. Величины внутренних углов $\omega_{i}, i=1, \ldots, K$, во всех сингулярных точках границы находятся в интервале $(0, \pi)$. 
ПРЕДПОЛОЖЕНИЕ $\mathrm{A}_{3}^{\prime \prime}$. Отображение $\psi_{0}$ принадлежсит классу $B_{p}^{2-1 / p}(\partial \Omega)$ при $p>2$. Семейство отображсений $\psi: B_{p}^{2-1 / p}(\partial \Omega) \times \mathbb{R}_{\varepsilon} \rightarrow \mathbb{R}$ является аналитическим по совокупности переменных и удовлетворяет начальному условию $\psi(0)=\psi_{0}$.

ТЕорема 2.2. При предположениях $\mathrm{A}_{1}, \mathrm{~A}_{2}^{\prime \prime}, \mathrm{A}_{3}^{\prime \prime}$ нелинейный оператор $P$ действует в пространствах

$$
P: W_{p}^{2}(\Omega) \times \mathbb{R} \times \mathbb{R}_{\varepsilon} \rightarrow L_{p}(\Omega) \times B_{p}^{2-1 / p}(\partial \Omega), \quad p>2,
$$

является аналитическим отображением и его производная Фреше

$$
P^{\prime}(w) h=\left(H^{\prime}(w) h,\left.h\right|_{\partial \Omega}\right)
$$

в каждой точке $w \in C^{3}(\bar{\Omega})$ является линейным фредгольмовым индекса 0 оператором $\left(P^{\prime}(w) \in \Phi_{0}\right)$.

ДокАЗАТЕЛьство. Приведем два самых существенных элемента в доказательстве теоремы 2.2. А именно, докажем действие нелинейного оператора $P$ в пространствах (2.7) и установим фредгольмовость (индекса 0) линейного оператора $P_{w}^{\prime}(w, \lambda, \mu)$, см. $(2.4),(2.5)$, в тех же пространствах.

1. Для доказательства первого факта воспользуемся теоремами вложения пространств Соболева. Нелинейньй оператор Плато $P(w, \lambda, \mu)($ см. $(2.1))$ содержит дифференциальньй оператор

$$
F(w, \lambda)=-\left(1+\nabla w^{2}\right)^{-3 / 2}\left(\Delta w+w_{y}^{2} w_{x x}-2 w_{x} w_{y} w_{x y}+w_{x}^{2} w_{y y}\right)-\lambda\left(w-w_{0}\right) .
$$

Пусть функция $w \in W_{p}^{2}(\Omega)$. Докажем, например, что $w_{x} w_{y} w_{x y} \in L_{p}(\Omega)$. Действительно, $w_{x}, w_{y} \in W_{p}^{1}(\Omega)$ и в силу включения

$$
W_{p}^{m}(\Omega) \cdot W_{p}^{m}(\Omega) \subset W_{p}^{m}(\Omega), \quad m \geqslant 1, \quad p \geqslant 2,
$$

имеем $w_{x} w_{y} \in W_{p}^{1}(\Omega)$. Функция $w_{x y} \in L_{p}(\Omega)$. Используя вложение

$$
i: W_{p}^{1}(\Omega) \rightarrow L_{\infty}(\Omega), \quad p>2
$$

и включение

$$
L_{\infty}(\Omega) \cdot L_{p}(\Omega) \subset L_{p}(\Omega), \quad p \geqslant 2,
$$

получаем необходимое нам включение

$$
W_{p}^{1}(\Omega) \cdot L_{p}(\Omega) \subset L_{p}(\Omega), \quad p>2 .
$$

Следовательно, элемент $w_{x} w_{y} w_{x y} \in L_{p}(\Omega)$.

2. Докажем второе утверждение. Соответствуюшая производная Фреше имеет следующий вид

$$
P_{w}^{\prime}(w, \lambda, \mu) h=\left(-A(w) h-\lambda h,\left.h\right|_{\partial \Omega}\right)
$$


(см. (2.4), (2.5)) и может быть представлена в форме

$$
P_{w}^{\prime}(w, \lambda, \mu) h=\left(L(w) h,\left.h\right|_{\partial \Omega}\right)+(M(w, \lambda) h, 0),
$$

где

$$
L(w) h=-\left(1+\nabla w^{2}\right)^{-3 / 2}\left(h_{x x}+h_{y y}\right) .
$$

При $w=0$, например, оператор $L(0) h=-\Delta h$. Фредгольмовость и индекс оператора $-\Delta$ на пространствах Соболева

$$
L(0): W_{p 0}^{m}(\Omega) \rightarrow W_{p}^{m-2}(\Omega),
$$

при $p=2$ и $m \geqslant 2$ хорошо известны. Его индекс $-K(m-2)$ отрицательньй при $m>2$ и равен 0 только при $m=2$. Здесь, символом $W_{20}^{m}(\Omega)$ обозначено подпространство функций с нулевыми следами на гранище.

Однако, при $p=m=2$ отсутствует действие нелинейного оператора $P$, см. включения (2.9), (2.10). Чтобы обеспечить одновременно действие нелинейного оператора Плато и фредгольмовость индекса 0 оператора Лапласа в одних и тех же пространствах Соболева, необходимо использовать случай $p>2$ и $m=2$.

Изоморфизм оператора $L(w)$ в пространствах (2.12) при $p>2$ и $m=2$ следует из работы В. А. Кондратьева [19] о разрешимости в этих пространствах соответствуюшей эллиптической краевой задачи.

Из того, что $B_{p}^{2-1 / p}(\partial \Omega)$ является пространством следов для $W_{p}^{2}(\Omega)$, следует: первый оператор в разложении (2.11) является изоморфизмом.

Из компактности вложения $i: W_{p}^{2}(\Omega) \rightarrow L_{p}(\Omega)$ следует, что второй оператор в разложении (2.11) является вполне непрерывным. Его добавление не влияет на фредгольмовость и индекс оператора $P_{w}^{\prime}(w, \lambda, \mu)$.

Пространство $W_{p}^{2}(\Omega)$, в котором мы будем искать решения $w(x, y)$ задачи $(1.1)-(1.2)$, вложено только в пространство $C(\bar{\Omega})$ и не вложено в пространство $C^{1}(\bar{\Omega})$. Следовательно, в случае области с кусочно-гладкой гранищей, производные $w_{x}, w_{y}, w_{x x}, w_{x y}, w_{y y}$ в задаче (1.1)-(1.2) будем понимать в смысле дифференцирования обобшенных функций.

\section{3. Необходимое и достаточное условия бифуркации в точке однократного вырождения}

В банаховом пространстве $W_{p}^{2}(\Omega), p>2$, рассмотрим конечномерное подпространство $N(w, \lambda)=\operatorname{Ker} P_{w}^{\prime}(w, \lambda, \mu)$ решений $h(x, y)$ следуюшей линейной задачи

$$
\begin{cases}A(w) h-\lambda h=0, & (x, y) \in \Omega \\ h=0, & (x, y) \in \partial \Omega .\end{cases}
$$

Лемма 3.1. Пусть выполнены предположсения $\mathrm{A}_{1}, \mathrm{~A}_{2}^{\prime \prime}, \mathrm{A}_{3}^{\prime \prime}$. Тогда, для бифуркации решений уравнения $P(w, \lambda, \mu)=(0,0)$ в точке $\left(w_{0}, \lambda_{0}, 0\right)$ необходимо, чтобьь

$$
\operatorname{dim} N\left(w_{0}, \lambda_{0}\right) \neq 0
$$


ДокаЗАтельСтво. Пусть $\operatorname{dim} N\left(w_{0}, \lambda_{0}\right)=0$. Тогда, линейный оператор $P_{w}^{\prime}\left(w_{0}, \lambda_{0}, 0\right)$ принадлежит классу $\Phi_{0}$ и будет линейным изоморфизмом пространств Соболева (2.7). Следовательно, по теореме о неявном отображении множество решений уравнения $P(w, \lambda, \mu)=(0,0)$ в окрестности точки $\left(w_{0}, \lambda_{0}, 0\right)$ состоит только из семейства $\left\{\left(w_{0}, \lambda, \mu\right): \lambda \in \mathbb{R}, \mu \in \mathbb{R}_{\varepsilon}\right\}$.

Заметим, что точки $\lambda_{0}$, в которых вьполнено условие (3.2), являются собственньми значениями линейного оператора $A\left(w_{0}\right)$ на пространстве функций $W_{p 0}^{2}(\Omega)$ с нулевыми граничными значениями. Из общей теории линейных эллиптических уравнений следует (см. [18]), что оператор $A\left(w_{0}\right)$ имеет только положительные собственные значения и первое из них однократно.

В качестве достаточного условия бифуркации при однократном вырождении дифференциала Фреше применим теорему Crandall-Rabinowitz (см. [20, теорема 1], или [21, теорема 3.2 .2$])$.

Tеорема [CR]. В банаховых пространствах X и Y рассмотрим уравнение с параметром $\lambda \in \mathbb{R}$

$$
P(w, \lambda)=0, \quad P: X \times \mathbb{R} \rightarrow Y, \quad P \in C^{p}, \quad p \geqslant 3,
$$

в малой окрестности $\left\|w-w_{0}\right\|_{X}<\varepsilon,\left|\lambda-\lambda_{0}\right|<\varepsilon$.

Пусть виполнень следующие условия:

1. $P\left(w_{0}, \lambda_{0}\right)=0$,

2. $P_{\lambda}^{\prime}\left(w_{0}, \lambda_{0}\right)=0$,

3. $\operatorname{dim} \operatorname{Ker} P_{w}^{\prime}\left(w_{0}, \lambda_{0}\right)=1, P_{w}^{\prime}\left(w_{0}, \lambda_{0}\right) e=0, e \neq 0$,

4. $\operatorname{codim} \operatorname{Im} P_{w}^{\prime}\left(w_{0}, \lambda_{0}\right)=1$,

5. $P_{\lambda \lambda}^{\prime \prime}\left(w_{0}, \lambda_{0}\right) \in \operatorname{Im} P_{w}^{\prime}\left(w_{0}, \lambda_{0}\right)$,

6. $P_{w \lambda}^{\prime \prime}\left(w_{0}, \lambda_{0}\right) e \notin \operatorname{Im} P_{w}^{\prime}\left(w_{0}, \lambda_{0}\right)$.

Тогда точка $\left(w_{0}, \lambda_{0}\right)$ является точкой бифуркачии и множество решений уравнения

$$
P(w, \lambda)=0
$$

в окрестности этой точки состоит из двух гладких кривых, которье могут быть заданы параметрически следующим образом

$$
\begin{aligned}
\Gamma_{1} \in C^{p-2}: \quad(w(\lambda), \lambda), & \left(w\left(\lambda_{0}\right), \lambda_{0}\right)=\left(w_{0}, \lambda_{0}\right), \\
\left(w^{\prime}(\lambda), 1\right), & \left(w^{\prime}\left(\lambda_{0}\right), 1\right)=(0,1), \\
\Gamma_{2} \in C^{p-2}: \quad(w(t), \lambda(t)), & (w(0), \lambda(0))=\left(w_{0}, \lambda_{0}\right), \\
\left(w^{\prime}(t), \lambda^{\prime}(t)\right), & \left(w^{\prime}(0), \lambda^{\prime}(0)\right)=\left(e, \lambda_{0}^{\prime}\right),
\end{aligned}
$$

$u$ которые пересекаются только в точке $\left(w_{0}, \lambda_{0}\right), t \in(-\varepsilon,+\varepsilon)$ - параметр.

Сначала исследуем возможность бифуркации по основному параметру $\lambda$.

Рассмотрим уравнение (2.1) при $\mu=0$. В этом случае применение теоремы [CR] к уравнению (2.1) дает следуюший результат. 


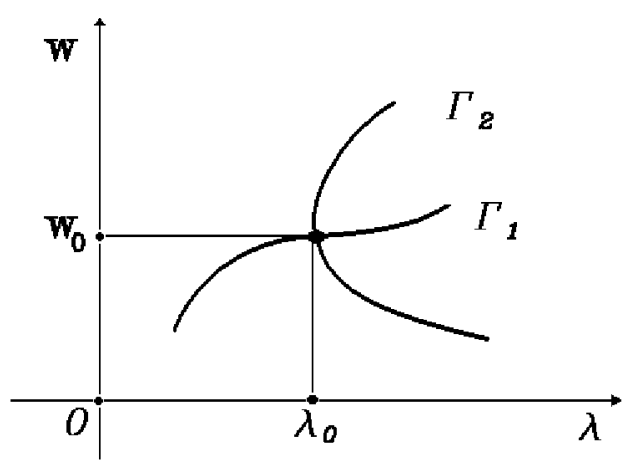

Рис. 2

ТЕОРемА 3.1. Пусть выполнены предположения $\mathrm{A}_{1}, \mathrm{~A}_{2}^{\prime \prime}, \mathrm{A}_{3}^{\prime \prime}$. Тогда, для бифуркачии решений уравнения $P(w, \lambda, 0)=(0,0)$ в точке $\left(w_{0}, \lambda_{0}\right)$ достаточно, чтоби

$$
\operatorname{dim} N\left(w_{0}, \lambda_{0}\right)=1
$$

ДокАЗАТЕЛЬСтво. Докажем, что выполнены условия 1-6, необходимые для применения теоремы $[\mathrm{CR}]$ о простой точке бифуркации. Первое условие теоремы $[\mathrm{CR}]$ выполнено в силу минимальности поверхности $w_{0}$, см. предположение $\mathrm{A}_{1}$ или (1.4). Третье условие - в силу предположения (3.3), а четвертое - в силу фредгольмовости индекса 0 оператора $P_{w}^{\prime}\left(w_{0}, \lambda_{0}, 0\right)$. Для проверки остальных условий вычислим необходимые нам производные $\Phi$ реше от оператора $P$ :

$$
\begin{gathered}
P_{\lambda}^{\prime}(w, \lambda)=\left(w_{0}-w, 0\right), \\
P_{w}^{\prime}(w, \lambda) h=\left(A(w) h-\lambda h,\left.h\right|_{\partial \Omega}\right), \\
P_{\lambda \lambda}^{\prime \prime}(w, \lambda)=(0,0), \\
P_{w \lambda}^{\prime \prime}(w, \lambda) h=(-h, 0) .
\end{gathered}
$$

Вьполнение условий 2 и 5 теоремы [CR] следует из того, что $P_{\lambda}^{\prime}\left(w_{0}, \lambda_{0}\right)=(0,0)$ и $P_{\lambda \lambda}^{\prime \prime}\left(w_{0}, \lambda_{0}\right)=(0,0)$.

Для проверки условия 6 рассмотрим ненулевой элемент $e \in N\left(w_{0}, \lambda_{0}\right)$. Он будет решением следуюшей краевой задачи

$$
\begin{cases}A\left(w_{0}\right) e-\lambda_{0} e=0, & (x, y) \in \Omega, \\ e=0, & (x, y) \in \partial \Omega .\end{cases}
$$

Предположим, что условие 6 теоремы [CR] не выполнено, т.е.

$$
P_{w \lambda}^{\prime \prime}\left(w_{0}, \lambda_{0}\right) e \in \operatorname{Im} P_{w}^{\prime}\left(w_{0}, \lambda_{0}\right)
$$

Это означает, что в пространстве $W_{p}^{2}(\Omega)$ существует элемент $h$ такой, что

$$
(-e, 0)=P_{w}^{\prime}\left(w_{0}, \lambda_{0}\right) h: \begin{cases}A\left(w_{0}\right) h-\lambda_{0} h=e, & \Omega, \\ h=0, & \partial \Omega .\end{cases}
$$


Тогда, из $(3.5),(2.4)$ и (3.7) получаем равенство

$$
-\iint_{\Omega} e^{2} d x d y=\iint_{\Omega}\left(A\left(w_{0}\right) h-\lambda_{0} h\right) e d x d y=\iint_{\Omega}\left(A\left(w_{0}\right) e-\lambda_{0} e\right) h d x d y=0
$$

которое противоречит предположению $e \neq 0$.

Следовательно, вьполнены все условия теоремы $[\mathrm{CR}]$, точка $\left(w_{0}, \lambda_{0}, 0\right)$ является точкой бифуркации и множество решений операторного уравнения $P(w, \lambda, 0)=$ $(0,0)$ в окрестности этой точки состоит из двух кривых (см. рис. 2 и теорему $[\mathrm{CR}])$.

Заметим, что к уравнению (2.1) не удается применить наиболее распространенные теоремы о бифуркациях в точках нечетнократного вырождения производной Фреше - теоремы Красносельского [22], Rabinowitz [23] и теорему Ize [24] (см., например, [21, теоремы 3.3.1, 3.4.1, 3.5.1]). Действительно, первая теорема применима к операторам вида $I-k$, где $k$ - компактный оператор и $I$ - тождественный. Вторая теорема применима к операторам вида $A-k$, где $A-$ линейньй фредгольмов оператор индекса 0 . В третьем случае нелинейность имеет порядок $O\left(|w|^{2}\right)$. Оператор Плато $P$ не удовлетворяет этим условиям.

В теореме 3.1 установлено достаточное условие для бифуркации минимальной поверхности раздела двух сред в капилляре при изменении параметра $\lambda$.

Исследуем, будет ли условие (3.3) достаточным для бифуркации по параметру $\mu$ в точке $\left(w_{0}, \lambda_{0}, 0\right)$ решений уравнения

$$
P\left(w, \lambda_{0}, \mu\right)=(0,0)
$$

Для проверки условий теоремы $[\mathrm{CR}]$ вычислим необходимые производные $\Phi$ реше от $P$ :

$$
\begin{gathered}
P_{\mu}^{\prime}(w, \lambda, \mu)=\left(0, \psi_{\mu}^{\prime}(\mu)\right), \\
P_{w}^{\prime}(w, \lambda, \mu) h=\left(A(w) h-\lambda h,\left.h\right|_{\partial \Omega}\right), \\
P_{\mu \mu}^{\prime \prime}(w, \lambda, \mu)=\left(0, \psi_{\mu \mu}^{\prime \prime}(\mu)\right), \\
P_{w \mu}^{\prime \prime}(w, \lambda, \mu) h=(0,0) .
\end{gathered}
$$

ЗАмечАниЕ. Теорема $[\mathrm{CR}]$ не обеспечивает достаточное условие бифуркации для уравнения (3.8), так как $P_{w \mu}^{\prime \prime}\left(w_{0}, \lambda_{0}, 0\right) e=0$ и невьполнено предположение 6 этой теоремы.

Пусть граница $\left.w\right|_{\partial \Omega}$ поверхности раздела претерпевает малые деформации одновременно с изменением главного параметра $\lambda$ вблизи найденной точки бифуркации $\lambda_{0}$

$$
\left.w\right|_{\partial \Omega}=\psi(\mu), \quad \mu=\mu(\lambda), \quad \mu\left(\lambda_{0}\right)=0
$$

Исследуем, будет ли условие (3.3) достаточным для бифуркации по параметру $\lambda$ в точке $\left(w_{0}, \lambda_{0}\right)$ решений уравнения

$$
P(w, \lambda, \mu(\lambda))=(0,0) .
$$


Tеорема 3.2. Рассмотрим задачу (1.1)-(1.2) и операторное уравнение (3.10) при предположениях $\mathrm{A}_{1}, \mathrm{~A}_{2}^{\prime \prime}$. Пусть отображсение $\psi(\mu), \psi: \mathbb{R}_{\varepsilon} \rightarrow$ $B_{p}^{2-1 / p}(\partial \Omega)$ и функция $\mu(\lambda)$ являются гладким класса $C^{p}, p \geqslant 3$, и для них выполнены следующие условия

1. $\psi(0)=\psi_{0}$,

2. $\mu\left(\lambda_{0}\right)=0$,

3. $\mu^{\prime}\left(\lambda_{0}\right)=0$,

4. $\psi^{\prime}(0) \in \Psi$ или $\mu^{\prime \prime}\left(\lambda_{0}\right)=0$, где $\Psi$ - подпространство в $B_{p}^{2-1 / p}(\partial \Omega)$, определенное по формуле

$$
\Psi=\left\{\psi: \psi=\left.h\right|_{\partial \Omega}, h \in W_{p}^{2}(\Omega), A\left(w_{0}\right) h-\lambda_{0} h=0\right\} .
$$

5. $\Pi y c m b \operatorname{dim} N\left(w_{0}, \lambda_{0}\right)=1$.

Тогда точка $\left(w_{0}, \lambda_{0}\right)$ является точкой бифуркачии в задаче (3.11) и множество решений в ее окрестности состоит из двух кривых (см. теоремy $[\mathrm{CR}])$.

ДокАЗАТЕЛЬСтво. Для проверки условий теоремы $[\mathrm{CR}]$ вычислим необходимые производные $Ф$ реше от $P$ :

$$
\begin{gathered}
P_{\lambda}^{\prime}(w, \lambda)=\left(\left(w-w_{0}\right), \psi^{\prime}(\mu) \mu^{\prime}(\lambda)\right), \\
P_{w}^{\prime}(w, \lambda) h=\left(A(w) h-\lambda h,\left.h\right|_{\partial \Omega}\right), \\
P_{\lambda \lambda}^{\prime \prime}(w, \lambda)=\left(0, \psi^{\prime \prime}(\mu)\left(\mu^{\prime}(\lambda)\right)^{2}+\psi^{\prime}(\mu) \mu^{\prime \prime}(\lambda)\right), \\
P_{w \lambda}^{\prime \prime}(w, \lambda) h=(-h, 0) .
\end{gathered}
$$

Вычислим необходимые производные Фреше в точке $\left(w_{0}, \lambda_{0}\right)$ :

$$
\begin{gathered}
P_{\lambda}^{\prime}\left(w_{0}, \lambda_{0}\right)=(0,0), \\
P_{w}^{\prime}\left(w_{0}, \lambda_{0}\right) h=\left(A\left(w_{0}\right) h-\lambda_{0} h,\left.h\right|_{\partial \Omega}\right), \\
P_{\lambda \lambda}^{\prime \prime}\left(w_{0}, \lambda_{0}\right)=\left(0, \psi^{\prime}(0) \mu^{\prime \prime}\left(\lambda_{0}\right)\right), \\
P_{w \lambda}^{\prime \prime}\left(w_{0}, \lambda_{0}\right) h=(-h, 0) .
\end{gathered}
$$

Проверка условий $1,3,4$ и 6 теоремы [CR] происходит точно также, как и в теореме 3.1. Условия 2 и 5 теоремы [CR] будут иметь следующий вид

$$
\begin{gathered}
P_{\lambda}^{\prime}\left(w_{0}, \lambda_{0}\right)=\left(0, \psi^{\prime}\left(\lambda_{0}\right)\right)=(0,0), \\
P_{\lambda \lambda}^{\prime \prime}\left(w_{0}, \lambda_{0}\right)=\left(0, \psi^{\prime}(0) \mu^{\prime \prime}\left(\lambda_{0}\right)\right) \in \operatorname{Im} P_{w}^{\prime}\left(w_{0}, \lambda_{0}\right) .
\end{gathered}
$$

Последнее из условий (3.15) означает: в пространстве $W_{p}^{2}(\Omega)$ существует элемент $h$ такой, что

$$
\begin{cases}A\left(w_{0}\right) h-\lambda_{0} h=0, & (x, y) \in \Omega, \\ h=\mu^{\prime \prime}\left(\lambda_{0}\right) \psi^{\prime}(0), & (x, y) \in \partial \Omega .\end{cases}
$$

Существование такого $h$ обеспечивается предположением 4 данной теоремы.

ЗАмЕчаниЕ. Третье из условий теоремы 3.2 означает, что бифуркация минимальной поверхности раздела $w_{0}(x, y)$, найденная в теореме 3.1 , сохранится, если деформация границы будет происходить медленнее, чем рост параметра $\lambda$. 


\section{4. Метод конечномерной редукции}

Для получения более обшей информации о возможных перестройках множества решений задачи (1.1)-(1.2) в окрестности простой точки бифуркации $\left(w_{0}, \lambda_{0}, 0\right)$ при наличии дополнительных возмушений необходимо использовать методы теории особенностей гладких отображений (см., например, [25]).

Для этого, во-первых, операторное уравнение (2.1) с фредгольмовым индекса 0 оператором в окрестности точки $\left(w_{0}, \lambda_{0}, 0\right)$ редуцируем к проблеме ветвления множества критических точек ключевой функции $\Phi(\xi, \lambda, \mu)$ с параметрами $\lambda$ и $\mu$

$$
\nabla_{\xi} \Phi(\xi, \lambda, \mu)=0
$$

которая определена локально в окрестности точки $\left(0, \lambda_{0}, 0\right)$ и

$$
\Phi: \mathbb{R}_{\varepsilon} \times\left(\lambda_{0}-\varepsilon, \lambda_{0}+\varepsilon\right) \times \mathbb{R}_{\varepsilon} \rightarrow \mathbb{R}, \quad \nabla_{\xi} \Phi\left(0, \lambda_{0}, 0\right)=0 .
$$

Существует много различных модификаций конечномерной редукции Ляпунова-Шмидта для вариационных задач. В данной работе будем придерживаться схемы Сапронова [26].

Рассмотрим случай однократного вырождения в точке $\left(w_{0}, \lambda_{0}, 0\right)$ дифференциала $\Phi$ реше $P_{w}^{\prime}\left(w_{0}, \lambda_{0}, 0\right) h=\left(F_{w}^{\prime}\left(w_{0}, \lambda_{0}\right) h,\left.h\right|_{\partial \Omega}\right)$ в задаче $(2.1)$. Пусть

$$
\operatorname{dim} N\left(w_{0}, \lambda_{0}\right)=1,
$$

и подпространство $N\left(w_{0}, \lambda_{0}\right)$ порождено элементом $e \neq 0$ таким, что

$$
P_{w}^{\prime}\left(w_{0}, \lambda_{0}, 0\right) e=0, \quad \iint_{\Omega} e^{2}(x, y) d x d y=1
$$

Тогда по классической схеме Ляпунова-Шмидта (см., например, [27]) от операторного уравнения (2.1) можно перейти к одномерному уравнению разветвления

$$
\phi(\xi, \lambda, \mu) \equiv \iint_{\Omega}\left(w(\xi, \lambda, \mu)-w_{0}\right) e d x d y-\xi=0,
$$

где функция разветвления $\phi(\xi, \lambda, \mu)$ определена локально

$$
\phi: \mathbb{R}_{\varepsilon} \times\left(\lambda_{0}-\varepsilon, \lambda_{0}+\varepsilon\right) \times \mathbb{R}_{\varepsilon} \rightarrow \mathbb{R}, \quad \phi\left(0, \lambda_{0}, 0\right)=0,
$$

и является достаточно гладкой (класса $\left.C^{\infty}\right)$, а отображение $w(\xi, \lambda, \mu)(x, y)$ задано неявно как решение следуюшей нелинейной краевой задачи в $\Omega$

$$
\left\{\begin{array}{l}
F(w, \lambda)-\left(\iint_{\Omega}\left(w-w_{0}\right) e d x d y-\xi\right) e=0 \\
\left.w\right|_{\partial \Omega}=\psi(\mu)
\end{array}\right.
$$


с параметрами $\xi, \lambda, \mu$, определенньми локально в окрестности точки $\left(0, \lambda_{0}, 0\right)$. Отображение

$$
\phi: \mathbb{R}_{\varepsilon} \times\left(\lambda_{0}-\varepsilon, \lambda_{0}+\varepsilon\right) \times \mathbb{R}_{\varepsilon} \rightarrow W_{p}^{2}(\Omega), \quad w\left(0, \lambda_{0}, 0\right)=w_{0},
$$

тоже принадлежит классу гладкости $C^{\infty}$.

Сушествование $w(\xi, \lambda, \mu)(x, y)$ следует из теоремы о неявном отображении. Действительно, задачу (4.4) запишем в виде операторного уравнения

$$
\widehat{P}(w, \lambda, \xi, \mu)=(0,0),
$$

где нелинейный оператор $\widehat{P}$ определен по формуле

$$
\widehat{P}(w, \lambda, \xi, \mu) \equiv\left(F(w, \lambda)-\left(\iint_{\Omega}\left(w-w_{0}\right) e d x d y-\xi\right) e,\left.w\right|_{\partial \Omega}-\psi(\mu)\right)
$$

и действует в пространствах Соболева

$$
\widehat{P}: W_{p}^{2}(\Omega) \times \mathbb{R} \times \mathbb{R}_{\varepsilon}^{2} \rightarrow B_{p}^{2-1 / p}(\partial \Omega), \quad \widehat{P}\left(w_{0}, \lambda_{0}, 0,0\right)=(0,0) .
$$

Тогда его дифференциал $\Phi$ реше в точке $\left(w_{0}, \lambda_{0}, 0\right)$

$$
\widehat{P}_{w}^{\prime}\left(w_{0}, \lambda_{0}, 0,0\right) h=\left(F_{w}^{\prime}\left(w_{0}, \lambda_{0}\right) h-\left(\iint_{\Omega} h e d x d y\right) e,\left.h\right|_{\partial \Omega}\right)
$$

будет изоморфизмом пространств Соболева и к уравнению (4.5) применима теорема о неявном отображении.

Согласно схеме в работе [26] множество решений уравнения разветвления $\phi(\xi, \lambda, \mu)=0$ в окрестности точки $\left(0, \lambda_{0}, 0\right)$ можно рассматривать как множество критических точек ключевой функции $\Phi(\xi, \lambda, \mu)$ с параметрами $\lambda, \mu$,

$$
\nabla_{\xi} \Phi(\xi, \lambda, \mu)=\phi(\xi, \lambda, \mu)
$$

которая может быть вычислена по формуле

$$
\Phi(\xi, \lambda, \mu)=E(w(\xi, \lambda, \mu), \lambda)+\frac{1}{2}\left(\iint_{\Omega}\left(w(\xi, \lambda, \mu)-w_{0}\right) e d x d y-\xi\right)^{2},
$$

где $w(\xi, \lambda, \mu)(x, y)$ - неявное отображение, найденное из уравнения (4.5).

Тогда исследование бифуркации решений уравнения (2.1) эквивалентно изучению перестройки множества критических точек ключевой функции [26] $\Phi_{0}(\xi)=$ $\Phi\left(\xi, \lambda_{0}, 0\right)$ при ее двупараметрической деформации

$$
\Phi_{0}(\xi)+\delta \Phi(\xi, \lambda, \mu)=0
$$

где $\delta \Phi(\xi, \lambda, \mu)=\Phi(\xi, \lambda, \mu)-\Phi\left(\xi, \lambda_{0}, 0\right)$ и $\delta \Phi\left(\xi, \lambda_{0}, 0\right)=0$. 
Естественен вопрос об устойчивых (находящихся в общем положении) перестройках множества критических точек функции $\Phi_{0}(\xi)$ при ее всевозможных деформациях. Для ответа на него (см. [25]) необходимо определить тип особенности критической точки $\xi_{0}=0$ функции $\Phi_{0}(\xi)$, коразмерность особенности $M$ (число Милнора) и вид миниверсальной деформации.

Tеорема 4.1. Рассмотрим задачу (1.1)-(1.2) при наиболее общих условиях $\mathrm{A}_{1}, \mathrm{~A}_{2}^{\prime \prime}, \mathrm{A}_{3}^{\prime \prime}$, при которых она әквивалентна операторному уравнению (2.1) в пространствах Соболева (2.7). Пусть $\left(w_{0}, \lambda_{0}, 0\right)$ - точка однократного вырождения дифференииала Фреше $P_{w}^{\prime}\left(w_{0}, \lambda_{0}, 0\right)$ в уравнении (2.1), m.е. $\operatorname{dim} N\left(w_{0}, \lambda_{0}\right)=1$, и подпространство $N\left(w_{0}, \lambda_{0}\right)$ порождено әлементом $e \neq 0$, удовлетворяющим условиям (4.2).

Тогда

1. Проблема бифуркации в точке $\left(w_{0}, \lambda_{0}, 0\right)$ решений уравнения (2.1) эквивалентна задаче (4.1) о ветвлении критических точек ключевой функции $\Phi(\xi, \lambda, \mu)$, определенной равенством (4.7) и операторныц уравнением (4.5).

2. Если функиии $w_{0}(x, y)$ и е $(x, y)$ таковы, что в каждой точке $(x, y) \in \Omega$ выполнено условие

$$
\nabla w_{0} \nabla e \neq 0,
$$

то ключевая функиия $\Phi_{0}(\xi)$, определенная равенством (4.8), в своей критической точке $\xi_{0}=0$ имеет особенность типа $\mathrm{A}_{2}$ ("складка") с коразмерностью (число Милнора) $M=1$.

3. Если функции $w_{0}(x, y)$ и е $(x, y)$ таковы, что в каждой точке $(x, y) \in \Omega$ выполнены условия

$$
\begin{gathered}
\nabla w_{0} \nabla e=0, \\
(\nabla e)^{2}+\nabla w_{0} \nabla w_{0}^{\prime \prime} \neq 0,
\end{gathered}
$$

где әлемент $w_{0}^{\prime \prime}$ является решением краевой задачи в области $\Omega$

$$
\left\{\begin{array}{l}
A\left(w_{0}\right) w_{0}^{\prime \prime}-\left(\iint_{\Omega} w_{0}^{\prime \prime} e d x d y\right) e-\lambda_{0} w_{0}^{\prime \prime}=-F_{0}^{(2)} e e \\
\left.w_{0}^{\prime \prime}\right|_{\partial \Omega}=0
\end{array}\right.
$$

правая часть которой определяется равенством

$$
F_{0}^{(2)} e e=3 \operatorname{div}\left(\left(1+\nabla w_{0}^{2}\right)^{-5 / 2}\left\{\nabla e^{2}+\nabla w_{0}^{2} \nabla e^{2}-\left(\nabla w_{0} \nabla e\right)^{2}\right\} \nabla w_{0}\right),
$$

то ключевая функиия $\Phi_{0}(\xi)$, определенная равенством (4.8), в своей критической точке $\xi_{0}=0$ имеет особенность типа $\mathrm{A}_{3}$ ("сборка") с коразмерностью (число Милнора) $M=2$.

4. Если функиии $w_{0}(x, y)$ и е $(x, y)$ таковы, что в каждой точке $(x, y) \in \Omega$ выполнены условия

$$
\begin{gathered}
\nabla w_{0} \nabla e=0, \\
(\nabla e)^{2}+\nabla w_{0} \nabla w_{0}^{\prime \prime}=0,
\end{gathered}
$$

то ключевая функиия $\Phi_{0}(\xi)$ в своей критической точке $\xi_{0}=0$ имеет особенность типа $\mathrm{A}_{k}$ c некоторьмм $k>3$. 
СЛЕДСТВИЕ 1. Из условий (4.10) u (4.2) следует, что если $w_{0}(x, y)=$ const - плоская поверхность раздела, направленная перпендикулярно ускорению $g$, то в однократных точках бифуркации $\left(w_{0}, \lambda_{0}, 0\right)$ всегда будет особенность muпа $\mathrm{A}_{3}$ ( "сборка").

СлЕДСТвИЕ 2. Если задачу (1.1)-(1.2) рассматривать в области $\Omega$ с гладкой граничей при предположсениях $\mathrm{A}_{1}, \mathrm{~A}_{2}^{\prime}, \mathrm{A}_{3}^{\prime}$, то для операторного уравнения (2.1) в пространствах Гёльдера (2.2) будет справедлива теорема 4.1 .

ДокАЗАТЕЛьство. Вычисление типа особенности построено на разложении функций и отображений в ряды Тейлора в точке бифуркации.

Рассмотрим случай $\xi_{0}=0$ и $\lambda \in\left(\lambda_{0}-\varepsilon, \lambda_{0}+\varepsilon\right)$ и $\mu \in \mathbb{R}_{\varepsilon}$. Из формулы (4.3) для функции $\phi(\xi, \lambda, \mu)$ следует, что

$$
\begin{aligned}
& \phi(0, \lambda, \mu)=\iint_{\Omega}\left(w(0, \lambda, \mu)-w_{0}\right) e d x d y, \\
& \phi_{\xi}^{\prime}(0, \lambda, \mu)=\iint_{\Omega} w_{\xi}^{\prime}(0, \lambda, \mu) e d x d y-1, \\
& \phi_{\xi}^{(n)}(0, \lambda, \mu)=\iint_{\Omega} w_{\xi}^{(n)}(0, \lambda, \mu) e d x d y, \quad n=2,3, \ldots
\end{aligned}
$$

Коэффициент $\omega^{(0)}=\omega(0, \lambda, \mu)$ находится из (4.4) или (4.5) при $\xi=0$

$$
\left(F\left(w^{(0)}, \lambda\right)-\left(\iint_{\Omega} w^{(0)} e d x d y-1\right) e,\left.w^{(0)}\right|_{\partial \Omega}\right)=(0, \psi(\mu)) .
$$

Для нахождения остальных коэффициентов $w^{(n)}=w_{\xi}^{(n)}(0, \lambda, \mu), n=1,2,3$, будем дифференцировать по $\xi$ в точке $\xi_{0}=0$ равенство (4.5). Получаем

$$
\begin{gathered}
\left(F^{(1)} w^{\prime}-\left(\iint_{\Omega} w^{\prime} e d x d y-1\right) e,\left.w^{\prime}\right|_{\partial \Omega}\right)=(0,0) \\
\left(F^{(1)} w^{\prime \prime}-\left(\iint_{\Omega} w^{\prime \prime} e d x d y\right) e+F^{(2)} w^{\prime} w^{\prime},\left.w^{\prime \prime}\right|_{\partial \Omega}\right)=(0,0) \\
\left(F^{(1)} w^{\prime \prime \prime}-\left(\iint_{\Omega} w^{\prime \prime \prime} e d x d y\right) e+3 F^{(2)} w^{\prime} w^{\prime \prime}+F^{(3)} w^{\prime} w^{\prime} w^{\prime},\left.w^{\prime \prime \prime}\right|_{\partial \Omega}\right)=(0,0),
\end{gathered}
$$

где символом $F^{(n)} h_{1} \ldots h_{n}=D_{w}^{(n)} F\left(w_{0}, \lambda\right) h_{1} \ldots h_{n}$ обозначен дифференциал ше от $F$ по переменной $w$ порядка $n$ в точке $\left(w_{0}, \lambda\right)$, вычисленньй на элементах $h_{1}, \ldots, h_{n}$.

Используя тот факт, что линейный оператор $\widehat{P}_{w}^{\prime}\left(w_{0}, \lambda, \mu, 0\right)$, определенный по правилу

$$
\widehat{P}_{w}^{\prime}\left(w_{0}, \lambda, \mu, 0\right) h=\left(A\left(w_{0}\right) h-\left(\iint_{\Omega} h e d x d y\right) e-\lambda h,\left.h\right|_{\partial \Omega}\right)
$$


является обратимьм при $\lambda \in\left(\lambda_{0}-\varepsilon, \lambda_{0}+\varepsilon\right)$ и $\mu \in \mathbb{R}_{\varepsilon}$ (изоморфизмом пространств Соболева), получаем рекуррентные формулы для $w^{\prime}, w^{\prime \prime}, w^{\prime \prime \prime}$ :

$$
\begin{aligned}
w^{\prime} & =\widehat{P}_{w}^{\prime}\left(w_{0}, \lambda, \mu, 0\right)^{-1}(-e, 0), \\
w^{\prime \prime} & =\widehat{P}_{w}^{\prime}\left(w_{0}, \lambda, \mu, 0\right)^{-1}\left(-F^{(2)} w^{\prime} w^{\prime}, 0\right), \\
w^{\prime \prime \prime} & =\widehat{P}_{w}^{\prime}\left(w_{0}, \lambda, \mu, 0\right)^{-1}\left(-3 F^{(2)} w^{\prime} w^{\prime \prime}-F^{(3)} w^{\prime} w^{\prime} w^{\prime}, 0\right) .
\end{aligned}
$$

Подставляя (4.17) в равенства (4.14), можно получить рекуррентные формулы первых коэффициентов ряда Тейлора для функции разветвления.

Формулы (4.14)-(4.17) можно сушественно упростить, если коэффициенты функции разветвления и коэффициенты $w_{0}^{(n)}=w_{\xi}^{(n)}\left(0, \lambda_{0}, 0\right), n=0,1,2,3$, вычислять только в одной точке $(\lambda, \mu)=\left(\lambda_{0}, 0\right)$.

Например,

$$
\begin{aligned}
w_{0}^{(0)} & =w_{0}, \\
w_{0}^{\prime} & =e,
\end{aligned}
$$

и элемент $w_{0}^{\prime \prime}$ является решением краевой задачи в области $\Omega$

$$
\left\{\begin{array}{l}
A\left(w_{0}\right) w_{0}^{\prime \prime}-\left(\iint_{\Omega} w_{0}^{\prime \prime} e d x d y\right) e-\lambda_{0} w_{0}^{\prime \prime}=-F_{0}^{(2)} e e \\
\left.w_{0}^{\prime \prime}\right|_{\partial \Omega}=0
\end{array}\right.
$$

Заметим, что в силу $(4.15)$ все функции $w^{(n)}(x, y)$ равны нулю на границе области $\Omega$, т.е. принадлежат подпространству $W_{p 0}^{2}(\Omega)$, на котором справедлива формула

$$
\iint_{\Omega} F_{0}^{(1)} h e d x d y=\iint_{\Omega} F_{0}^{(1)} e h d x d y=0
$$

где $F_{0}^{(1)}=A\left(w_{0}\right)-\lambda_{0} I$ и $A\left(w_{0}\right) h=-\operatorname{div}\left(\vec{p}\left(w_{0}\right) \nabla h\right)-$ дифференшиальньй эллиптический оператор, определенньй по формуле (2.4). Условие (4.20) означает, что для отображения $F_{0}^{(1)}: W_{p 0}^{2}(\Omega) \rightarrow L_{p}(\Omega)$ подпространство $\operatorname{Im} F_{0}^{(1)}$ является ортогональньм к подпространству $\operatorname{Ker} F_{0}^{(1)}$ относительно скалярного произведения в более широком гильбертовом пространстве $L_{2}(\Omega)$, для которого справедливы всюду плотные вложения $L_{2}(\Omega) \supset L_{p}(\Omega) \supset W_{p 0}^{2}(\Omega)$.

Тогда, в равенствах $(4.15)$ при $(\lambda, \mu)=\left(\lambda_{0}, 0\right)$ умножим первые компоненты на функцию $e(x, y)$ и проинтегрируем по области $\Omega$. Получим следуюшие формулы.

$$
\begin{gathered}
\iint_{\Omega} w_{0}^{\prime} e d x d y=1 \\
\iint_{\Omega} w_{0}^{\prime \prime} e d x d y=\iint_{\Omega} F_{0}^{(2)} w_{0}^{\prime} w_{0}^{\prime} e d x d y \\
\iint_{\Omega} w_{0}^{\prime \prime \prime} e d x d y=\iint_{\Omega}\left(3 F_{0}^{(2)} w_{0}^{\prime} w_{0}^{\prime \prime}+F_{0}^{(3)} w_{0}^{\prime} w_{0}^{\prime} w_{0}^{\prime}\right) e d x d y .
\end{gathered}
$$


Из представления (1.1) функционала $E(w, \lambda)$ и формулы $(1.10)$ для его первой вариации следует, что

$$
D_{w}^{\prime} E(w, \lambda) h=\iint_{\Omega} F(w, \lambda) h d x d y
$$

для любого $h \in W_{p 0}^{2}(\Omega)$. Дифференцируя по $w$ равенство (4.22), получаем формулы

$$
D_{w}^{(n+1)} E(w, \lambda) h_{1} \ldots h_{n} h_{n+1}=\iint_{\Omega}\left(D_{w}^{(n)} F(w, \lambda) h_{1} \ldots h_{n}\right) h_{n+1} d x d y
$$

справедливые при $n=1,2,3, \ldots$ и для $h_{1}, \ldots, h_{n+1} \in W_{p 0}^{2}(\Omega)$.

Подставляя $(4.18),(4.21)$ и (4.23) в формулы (4.14) получаем формулы первых коэффициентов ряда Тейлора ключевой функции

$$
\begin{aligned}
\Phi_{\xi}^{\prime}\left(0, \lambda_{0}, 0\right) & =\phi\left(0, \lambda_{0}, 0\right)=0 \\
\Phi_{\xi}^{\prime \prime}\left(0, \lambda_{0}, 0\right) & =\phi_{\xi}^{\prime}\left(0, \lambda_{0}, 0\right)=E_{0}^{(3)} e e e \\
\Phi_{\xi}^{\prime \prime \prime}\left(0, \lambda_{0}, 0\right) & =\phi_{\xi}^{\prime \prime}\left(0, \lambda_{0}, 0\right)=3 E_{0}^{(3)} e e w_{0}^{\prime \prime}+E_{0}^{(4)} \text { eeee },
\end{aligned}
$$

где $E_{0}^{(n)} h_{1} \ldots h_{n}=D_{w}^{(n)} E\left(w_{0}, \lambda_{0}\right) h_{1} \ldots h_{n}$ и элемент $w_{0}^{\prime \prime}$ является решением краевой задачи (4.19).

Вычислим первые производные $\Phi$ реше $E^{(n)}, n=1,2,3$, функционала $E$ по переменной $w$ :

$$
\begin{aligned}
E(w, \lambda)= & \iint_{\Omega}\left(\left(1+\nabla w^{2}\right)^{1 / 2}-\frac{\lambda}{2}\left(w-w_{0}\right)^{2}\right) d x d y, \\
E^{(1)}(w, \lambda) h= & \iint_{\Omega}\left(\left(1+\nabla w^{2}\right)^{-1 / 2}(\nabla w \nabla h)-\lambda\left(w-w_{0}\right) h\right) d x d y, \\
E^{(2)}(w, \lambda) h_{1} h_{2}= & \iint_{\Omega}\left(1+\nabla w^{2}\right)^{-3 / 2}\left(\left\{\left(1+\nabla w^{2}\right)\left(\nabla h_{1} \nabla h_{2}\right)\right.\right. \\
& \left.\left.-\left(\nabla w \nabla h_{1}\right)\left(\nabla w \nabla h_{2}\right)\right\}-\lambda h^{2}\right) d x d y, \\
E^{(3)}(w, \lambda) h_{1} h_{2} h_{3}= & \iint_{\Omega}\left(1+\nabla w^{2}\right)^{-5 / 2}\left(3\left(\nabla w \nabla h_{1}\right)\left(\nabla w \nabla h_{2}\right)\left(\nabla w \nabla h_{3}\right)\right. \\
& -\left(1+\nabla w^{2}\right)\left\{\left(\nabla w \nabla h_{1}\right)\left(\nabla h_{2} \nabla h_{3}\right)+\left(\nabla w \nabla h_{2}\right)\left(\nabla h_{1} \nabla h_{3}\right)\right. \\
& \left.\left.+\left(\nabla w \nabla h_{3}\right)\left(\nabla h_{1} \nabla h_{2}\right)\right\}\right) d x d y .
\end{aligned}
$$

Для вариации $\delta w=h$ аргумента $w$ вычислим первые вариации функционала $\delta^{n} E=D^{(n)} E(w, \lambda) h, \ldots, h, n=2,3,4$,

$$
\begin{aligned}
& \delta^{2} E=\iint_{\Omega}\left(1+\nabla w^{2}\right)^{-3 / 2}\left\{\nabla h^{2}+\nabla w^{2} \nabla h^{2}-(\nabla w \nabla h)^{2}-\lambda h^{2}\right\} d x d y \\
& \delta^{3} E=-3 \iint_{\Omega}\left(1+\nabla w^{2}\right)^{-5 / 2}(\nabla w \nabla h)\left\{\nabla h^{2}+\nabla w^{2} \nabla h^{2}-(\nabla w \nabla h)^{2}\right\} d x d y \\
& \delta^{4} E=-3 \iint_{\Omega}\left(1+\nabla w^{2}\right)^{-7 / 2}\left\{\nabla h^{2}+\nabla w^{2} \nabla h^{2}-(\nabla w \nabla h)^{2}\right\} \\
& \quad \times\left\{\nabla h^{2}+\nabla w^{2} \nabla h^{2}-5(\nabla w \nabla h)^{2}\right\} d x d y
\end{aligned}
$$


Для вычисления $F^{(2)} h h$ воспользуемся представлением (4.26) для третьей вариации $\delta^{3} E$ и формулой (4.23). Получаем

$$
\begin{aligned}
\delta^{3} E & =3 \iint_{\Omega} \operatorname{div}\left(\left(1+\nabla w^{2}\right)^{-5 / 2}\left\{\nabla h^{2}+\nabla w^{2} \nabla h^{2}-(\nabla w \nabla h)^{2}\right\} \nabla w\right) h d x d y \\
& =\iint_{\Omega}\left(F^{(2)} h h\right) h d x d y
\end{aligned}
$$

и, следовательно,

$$
F_{0}^{(2)} e e=3 \operatorname{div}\left(\left(1+\nabla w_{0}^{2}\right)^{-5 / 2}\left\{\nabla e^{2}+\nabla w_{0}^{2} \nabla e^{2}-\left(\nabla w_{0} \nabla e\right)^{2}\right\} \nabla w_{0}\right) .
$$

Для вычисления коэффициентов ключевой функции подставим найденные формулы в (4.24) и (4.19). Получаем окончательные формулы:

$$
\begin{aligned}
& \Phi_{\xi}^{\prime}\left(0, \lambda_{0}, 0=\right. \phi\left(0, \lambda_{0}, 0\right)=0 \\
& \Phi_{\xi}^{\prime \prime}\left(0, \lambda_{0}, 0\right)= \phi^{\prime}\left(0, \lambda_{0}, 0\right)=0 \\
& \Phi_{\xi}^{\prime \prime \prime}\left(0, \lambda_{0}, 0\right)= \phi_{\xi}^{\prime \prime}\left(0, \lambda_{0}, 0\right) \\
&=-3 \iint_{\Omega}\left(1+\nabla w_{0}^{2}\right)^{-5 / 2}\left(\nabla w_{0} \nabla e\right) \\
& \quad \times\left\{\nabla e^{2}+\nabla w_{0}^{2} \nabla e^{2}-\left(\nabla w_{0} \nabla e\right)^{2}\right\} d x d y \\
& \Phi_{\xi}^{(4)}\left(0, \lambda_{0}, 0\right)= \phi_{\xi}^{\prime \prime \prime}\left(0, \lambda_{0}, 0\right) \\
&=-3 \iint_{\Omega}\left(1+\nabla w_{0}^{2}\right)^{-7 / 2}\left(3\left(1+\nabla w_{0}^{2}\right)\left(\nabla w_{0} \nabla e\right)^{2}\left(\nabla w_{0} \nabla w_{0}^{\prime \prime}\right)\right. \\
&-\left(1+\nabla w_{0}^{2}\right)^{2}\left\{2\left(\nabla w_{0} \nabla e\right)\left(\nabla e \nabla w_{0}^{\prime \prime}\right)+\left(\nabla w_{0} \nabla w_{0}^{\prime \prime}\right) \nabla e^{2}\right\} \\
&-\left\{\nabla e^{2}+\nabla w_{0}^{2} \nabla e^{2}-\left(\nabla w_{0} \nabla e\right)^{2}\right\} \\
&\left.\times\left\{\nabla e^{2}+\nabla w_{0}^{2} \nabla e^{2}-5\left(\nabla w_{0} \nabla e\right)^{2}\right\}\right) d x d y,
\end{aligned}
$$

где элемент $w_{0}^{\prime \prime}$ является решением задачи (4.19) с правой частью (4.27).

Сделаем дополнительное предположение, что

$$
\nabla w_{0} \nabla e \equiv 0
$$

тогда коэффищиенты ряда Тейлора ключевой функции вычисляются по более простым формулам

$$
\begin{aligned}
\Phi_{\xi}^{\prime}\left(0, \lambda_{0}, 0\right) & =\phi\left(0, \lambda_{0}, 0\right)=0 \\
\Phi_{\xi}^{\prime \prime \prime}\left(0, \lambda_{0}, 0\right) & =\phi_{\xi}^{\prime \prime}\left(0, \lambda_{0}, 0\right)=0 \\
\Phi_{\xi}^{(4)}\left(0, \lambda_{0}, 0\right) & =\phi_{\xi}^{\prime \prime \prime}\left(0, \lambda_{0}, 0\right) \\
& =3 \iint_{\Omega}\left(1+\nabla w_{0}^{2}\right)^{-3 / 2} \nabla e^{2}\left(\nabla w_{0} \nabla w_{0}^{\prime \prime}+\nabla e^{2}\right) d x d y
\end{aligned}
$$

где $w_{0}^{\prime \prime}$ - решение линейной краевой задачи (4.19) с правой частью

$$
F_{0}^{(2)} e e=3 \operatorname{div}\left(\left(1+\nabla w_{0}^{2}\right)^{-3 / 2} \nabla e^{2} \nabla w_{0}\right) .
$$


В случае, если $w_{0}(x, y) \equiv$ const (плоская минимальная поверхность, направленная перпендикулярно ускорению $g$ ), имеем $\nabla w_{0} \equiv 0$ и, следовательно,

$$
\begin{aligned}
\Phi_{\xi}^{\prime}\left(0, \lambda_{0}, 0\right) & =\phi\left(0, \lambda_{0}, 0\right)=0, \\
\Phi_{\xi}^{\prime \prime}\left(0, \lambda_{0}, 0\right) & =\phi_{\xi}^{\prime}\left(0, \lambda_{0}, 0\right)=0, \\
\Phi_{\xi}^{\prime \prime \prime}\left(0, \lambda_{0}, 0\right) & =\phi_{\xi}^{\prime \prime}\left(0, \lambda_{0}, 0\right)=0, \\
\Phi_{\xi}^{(4)}\left(0, \lambda_{0}, 0\right) & =\phi_{\xi}^{\prime \prime \prime}\left(0, \lambda_{0}, 0\right)=3 \iint_{\Omega}|\nabla e|^{4} d x d y>0,
\end{aligned}
$$

критическая точка $\xi_{0}=0$ ключевой функции $\Phi_{0}(\xi)$ будет иметь особенность типа $\mathrm{A}_{3}$ ("сборка") с коразмерностью $M=2$.

Теорема 4.1 доказана.

Tеорема [CR] (M. G. Crandall, P.H. Rabinowitz), которая дает достаточное условие бифуркации в данной работе, получена с использованием леммы Морса и конечномерной редукции для фредгольмовых индекса 0 отображений банаховых пространств. В ней не содержится информация о расположении второй ветви решений в зависимости от параметра $\lambda$ и о количестве решений при $\lambda<\lambda_{0}$ и при $\lambda>\lambda_{0}$.

Возможны следуюшие ситуации:

1) совпадение в точке $\left(w_{0}, \lambda_{0}\right)$ двух ветвей решений $w_{0}(\lambda) \equiv w_{0}$ и $w_{1}(\lambda)$;

$2)$ слияние в точке $\left(w_{0}, \lambda_{0}\right)$ трех семейств решений $w_{0}(\lambda) \equiv w_{0}, w_{1}(\lambda)$ и $w_{2}(\lambda)$ в одно семейство $w_{0}(\lambda) \equiv w_{0}$.

Исследование бифуркаций в параметрической проблеме Плато [15] показало, что возможны обе ситуации. Для их распознавания необходимо дополнительное исследование задачи (1.1)-(1.2) и операторного уравнения (2.1). Оказалось, что расположение ветвей и их первая асимптотика по $\lambda$ зависят от коэффицциентов ключевой функции $\Phi_{\xi}^{(k)}\left(0, \lambda_{0}, 0\right), k=2,3, \ldots$, и, следовательно, от типа особенности ее критической точки $\xi_{0}=0$. Будет справедлива следуюшая теорема.

Теорема 4.2. Рассмотрим задачу (1.1)-(1.2) при наиболее общих условиях $\mathrm{A}_{1}, \mathrm{~A}_{2}^{\prime \prime}, \mathrm{A}_{3}^{\prime \prime}$, при которых она әквивалентна операторному уравнению (2.1) в пространствах Соболева (2.7). Пусть $\left(w_{0}, \lambda_{0}, 0\right)-$ точка однократного вырождения дифференииала Фреше $P_{w}^{\prime}\left(w_{0}, \lambda_{0}, 0\right)$ в уравнении $(2.1)$, m.е. $\operatorname{dim} N\left(w_{0}, \lambda_{0}\right)=1$, и подпространство $N\left(w_{0}, \lambda_{0}\right)$ породсдено әлементом e $\neq 0$, удовлетворяющим условиям

$$
P_{w}^{\prime}\left(w_{0}, \lambda_{0}, 0\right) e=0, \quad \iint_{\Omega} e^{2}(x, y) d x d y=1 .
$$

Тогда точка $\left(w_{0}, \lambda_{0}\right)$ является точкой бифуркации для операторного уравнения (2.1) и множсество решений в ее окрестности состоит из двух кривых $\Gamma_{1}=\left\{\left(w_{0}, \lambda\right): \lambda \in \mathbb{R}\right\}$ и $\Gamma_{2}$, пересекающихся трансверсально (см. теоремы $[\mathrm{CR}]$ u 3.1).

1. Если ключевая функиия $\Phi_{0}(\xi)$, определенная равенством (4.8), в своей критической точке $\xi_{0}=0$ имеет особенность типа $\mathrm{A}_{2}$ ( "складка"), см. теорему 4.1, то имеет место бифуркация, изображенная на рис. 3а, и семейство 
$\Gamma_{2}$ может быть параметризовано следующим образом:

$$
\Gamma_{2}:\left\{\begin{array}{l}
w_{2}(\lambda)=w_{0}+\frac{e}{\lambda_{1}^{*}}\left(\lambda-\lambda_{0}\right)+o\left(\lambda-\lambda_{0}\right), \\
\lambda \in\left(\lambda_{0}-\varepsilon, \lambda_{0}+\varepsilon\right)
\end{array}\right.
$$

$2 \partial e$

$$
\lambda_{1}^{*}=\frac{1}{2} \Phi_{\xi}^{\prime \prime \prime}\left(0, \lambda_{0}, 0\right) \neq 0
$$

см. формульц (4.28).

2. Если ключевая функиия $\Phi_{0}(\xi)$, определенная равенством (4.8), в своей критической точке $\xi_{0}=0$ имеет особенность типа $\mathrm{A}_{3}$ ("сборка"), см. теорему 4.1, то имеет место бифуркация, изображсенная на рис. $3 \mathrm{~b}$ или 3c, и семейство $\Gamma_{2}$ состоит из двух ветвей

$$
\Gamma_{2}:\left\{\begin{array}{l}
w_{2}^{ \pm}(\lambda)=w_{0} \pm e\left(\frac{\lambda-\lambda_{0}}{\lambda_{2}^{*}}\right)^{1 / 2}+o\left|\lambda-\lambda_{0}\right|^{1 / 2} \\
\lambda \in\left[\lambda_{0}, \lambda_{0}+\varepsilon\right), \text { ecлu } \lambda_{2}^{*}>0 \\
\lambda \in\left(\lambda_{0}-\varepsilon, \lambda_{0}\right], \text { ecлu } \lambda_{2}^{*}<0
\end{array}\right.
$$

$2 \partial e$

$$
\lambda_{2}^{*}=\frac{1}{3 !} \Phi_{\xi}^{(4)}\left(0, \lambda_{0}, 0\right) \neq 0,
$$

см. формульц (4.28), (4.19) и (4.27).

$$
P(w, \lambda)=0
$$

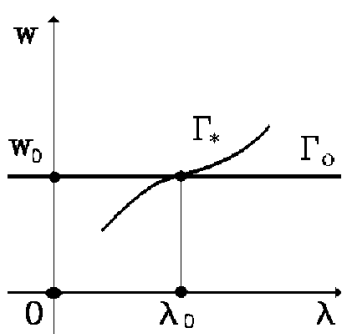

Рис. 3a

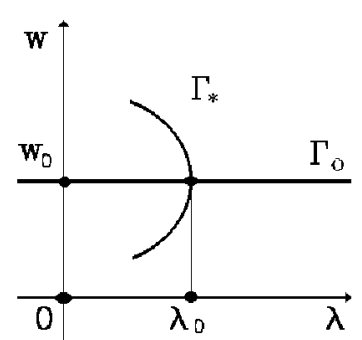

Рис. 3b

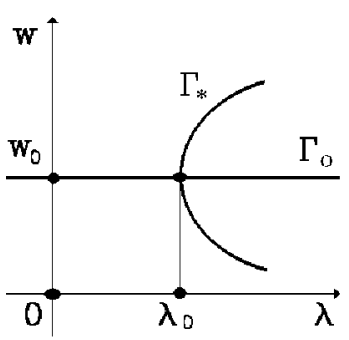

Рис. 3с

ДокАЗАТЕЛЬСтво. Из теоремы $[\mathrm{CR}]$ следует, что второе семейство решений, которое пересекает $\Gamma_{1}=\left\{\left(w_{0}, \lambda\right): \lambda \in \mathbb{R}\right\}$ в точке $\left(w_{0}, \lambda_{0}\right)$, может быть задано параметром $t \in(-\varepsilon, \varepsilon)$

$$
\Gamma_{2}:\left\{\begin{array}{l}
w(t)=w_{0}+w_{0}^{\prime} t+\frac{1}{2} w_{0}^{\prime \prime} t^{2}+\cdots \\
\lambda(t)=\lambda_{0}+\lambda_{0}^{\prime} t+\frac{1}{2} \lambda_{0}^{\prime \prime} t^{2}+\cdots
\end{array}\right.
$$


Подставим параметрическое представление (4.38) кривой $\Gamma_{2}$ в операторное уравнение (2.1) при $\mu=0$. Получим тождество

$$
\left(F(w(t), \lambda(t)),\left.w(t)\right|_{\partial \Omega}-\psi_{0}\right)=(0,0), \quad t \in(-\varepsilon,+\varepsilon)
$$

Заметим, что дифференциальный оператор $F$ имеет следуюший вид

$$
\begin{aligned}
F(w, \lambda) & =H(w)-\lambda\left(w-w_{0}\right), \\
F_{w}^{\prime}(w, \lambda) & =A(w)-\lambda I, \\
F_{w}^{(n)}(w, \lambda) & =H_{w}^{(n)}(w), \quad n=2,3, \ldots,
\end{aligned}
$$

см. формулы $(0.1),(1.3)$ и (2.4).

С учетом (4.40) продифференцируем тождество (4.39) по $t$ в точке $t_{0}=0$ :

$$
\begin{gathered}
\left(A(w) w^{\prime}-\lambda^{\prime}\left(w-w_{0}\right)-\lambda w^{\prime},\left.w^{\prime}\right|_{\partial \Omega}\right)=(0,0), \\
\left(A_{0} w_{0}^{\prime}-\lambda_{0} w_{0}^{\prime},\left.w_{0}^{\prime}\right|_{\partial \Omega}\right)=(0,0) .
\end{gathered}
$$

Решение $w_{0}^{\prime}=C e$ линейной задачи (4.41) может быть выбрано с точностью до константы $C \in \mathbb{R}$. Это свидетельствует о возможности нескольких параметризаций кривой $\Gamma_{2}$. В силу теорем $[\mathrm{CR}]$ и 3.1 от кривой $\Gamma_{1}$ в точке бифуркации $\left(w_{0}, \lambda_{0}\right)$ ответвляется единственное семейство решений $\Gamma_{2}$. Следовательно, его асимптотика по основному параметру $\lambda$ не зависит от выбора константы и способа параметризации кривой $\Gamma_{2}$. Выберем $w_{0}^{\prime}=e$.

После второго дифференцирования по $t$ получим

$$
\begin{gathered}
\left(F^{(2)}\left(w^{\prime}\right)^{2}+A(w) w^{\prime \prime}-\lambda^{\prime \prime}\left(w-w_{0}\right)-2 \lambda^{\prime} w^{\prime}-\lambda w^{\prime \prime},\left.w^{\prime \prime}\right|_{\partial \Omega}\right)=(0,0) \\
\left(A_{0} w_{0}^{\prime \prime}-\lambda_{0} w_{0}^{\prime \prime},\left.w_{0}^{\prime \prime}\right|_{\partial \Omega}\right)=\left(2 \lambda_{0}^{\prime} e-F_{0}^{(2)} e e, 0\right) .
\end{gathered}
$$

Задача (4.42) разрешима только при $\left(2 \lambda_{0}^{\prime} e-F_{0}^{(2)} e e\right) \in \operatorname{Im} F_{0}^{(1)}$, что обеспечивается условием

$$
\iint_{\Omega}\left(2 \lambda_{0}^{\prime} e-F_{0}^{(2)} e e\right) e d x d y=0
$$

Из формулы (4.42) при

$$
\lambda_{0}^{\prime}=\frac{1}{2} \iint_{\Omega}\left(F_{0}^{(2)} e e\right) e d x d y=\frac{1}{2} E_{0}^{(3)} e e e=\frac{1}{2} \Phi_{\xi}^{\prime \prime \prime}\left(0, \lambda_{0}, 0\right)
$$

находится элемент $w_{0}^{\prime \prime}$.

Если ключевая функция $\Phi_{0}(\xi)=\Phi\left(\xi, \lambda_{0}, 0\right)$ имеет в точке $\xi_{0}=0$ особенность типа $A_{2}$, то $\lambda_{0}^{\prime}=\frac{1}{2} \Phi_{\xi}^{\prime \prime \prime}\left(0, \lambda_{0}, 0\right) \neq 0$. Тогда в параметрическом задании $(4.38)$ кривой $\Gamma_{2}$ можно исключить $t$ следуюшим способом:

$$
\Gamma_{2}:\left\{\begin{array}{l}
t=\frac{\lambda-\lambda_{0}}{\lambda_{0}^{\prime}}+o\left(\lambda-\lambda_{0}\right), \\
w(\lambda)=w_{0}+\frac{e}{\lambda_{0}^{\prime}}\left(\lambda-\lambda_{0}\right)+o\left(\lambda-\lambda_{0}\right), \\
\lambda \in\left(\lambda_{0}-\varepsilon, \lambda_{0}+\varepsilon\right) .
\end{array}\right.
$$


Если ключевая функция $\Phi_{0}(\xi)$ имеет в точке $\xi_{0}=0$ особенность типа $\mathrm{A}_{3}$, то $\lambda_{0}^{\prime}=\frac{1}{2} \Phi_{\xi}^{\prime \prime \prime}\left(0, \lambda_{0}, 0\right)=0$ и тождество (4.39) необходимо третий раз продифференцировать по $t$. Получаем

$$
\begin{aligned}
& \left(F^{(3)}\left(w^{\prime}\right)^{3}+3 F^{(2)} w^{\prime} w^{\prime \prime}+A(w) w^{\prime \prime \prime}\right. \\
& \left.\quad-\lambda^{\prime \prime \prime}\left(w-w_{0}\right)-3 \lambda^{\prime \prime} w^{\prime}-3 \lambda^{\prime} w^{\prime \prime}-\lambda w^{\prime \prime \prime},\left.w^{\prime \prime \prime}\right|_{\partial \Omega}-\psi^{\prime \prime}\right)=(0,0) \\
& \quad\left(A_{0} w_{0}^{\prime \prime \prime}-\lambda_{0} w_{0}^{\prime \prime \prime},\left.w_{0}^{\prime \prime \prime}\right|_{\partial \Omega}\right)=\left(3 \lambda_{0}^{\prime \prime} e-F_{0}^{(3)} e^{3}-3 F_{0}^{(2)} e w_{0}^{\prime \prime}, 0\right)
\end{aligned}
$$

Задача (4.46) разрешима только при $\left(3 \lambda_{0}^{\prime \prime} e-F_{0}^{(3)} e^{3}-3 F_{0}^{(2)} e w_{0}^{\prime \prime}\right) \in \operatorname{Im} F_{0}^{(1)}$, что обеспечивается условием

$$
\iint_{\Omega}\left(3 \lambda_{0}^{\prime \prime} e-F_{0}^{(3)} e^{3}-3 F_{0}^{(2)} e w_{0}^{\prime \prime}\right) e d x d y=0
$$

Из формулы (4.46) при

$$
\begin{aligned}
\lambda_{0}^{\prime \prime} & =\frac{1}{3} \iint_{\Omega}\left(F_{0}^{(3)} e e e\right) e d x d y+\iint_{\Omega}\left(F_{0}^{(2)} e w_{0}^{\prime \prime}\right) e d x d y \\
& =\frac{1}{3} E_{0}^{(4)} \text { eeee }+E_{0}^{(3)} e e w_{0}^{\prime \prime}=\frac{1}{3} \Phi_{\xi}^{(4)}\left(0, \lambda_{0}, 0\right)
\end{aligned}
$$

находится элемент $w_{0}^{\prime \prime \prime}$.

Если ключевая функция $\Phi_{0}(\xi)$ имеет в точке $\xi_{0}=0$ особенность типа $\mathrm{A}_{3}$, то $\lambda_{0}^{\prime \prime}=\frac{1}{3} \Phi_{\xi}^{(4)}\left(0, \lambda_{0}, 0\right) \neq 0$. Тогда в параметрическом задании $(4.38)$ кривой $\Gamma_{2}$ можно исключить $t$ следующим способом:

$$
\Gamma_{2}:\left\{\begin{array}{l}
t^{2}=\frac{2\left(\lambda-\lambda_{0}\right)}{\lambda_{0}^{\prime \prime}}+o\left(\lambda-\lambda_{0}\right), \\
w(\lambda)=w_{0}+e \sqrt{\frac{2\left(\lambda-\lambda_{0}\right)}{\lambda_{0}^{\prime \prime}}}+o\left|\lambda-\lambda_{0}\right|^{1 / 2}, \\
\left|\lambda-\lambda_{0}\right|<\varepsilon .
\end{array}\right.
$$

Заметим, что

$$
\lambda_{1}^{*}=\lambda_{0}^{\prime}=\frac{1}{2} \Phi_{\xi}^{\prime \prime}\left(0, \lambda_{0}, 0\right), \quad \lambda_{2}^{*}=\frac{1}{2} \lambda_{0}^{\prime \prime \prime}=\frac{1}{3 !} \Phi_{\xi}^{(4)}\left(0, \lambda_{0}, 0\right)
$$

Теорема 4.2 доказана. 


\section{5. Исследование бифуркаций для капилляров с круглым и квадратным сечениями}

Исследуем краевую задачу (1.1)-(1.2) и операторное уравнение (2.1) при предположении, что область $\Omega$ (поперечное сечение капилляра) - круг радиуса 1 $\Omega=\left\{(x, y): x^{2}+y^{2}<1\right\}$, а граница поверхности раздела зафиксирована на боковой поверхности капилляра и задана функцией $\psi_{0} \equiv 0$. Тогда $w_{0}(x, y) \equiv 0-$ минимальная поверхность раздела при отсутствии гравитации $(\lambda=0)$.

Найдем значения параметра $\lambda \in \mathbb{R}$ при которых выполнено необходимое условие бифуркации (3.2) в лемме 3.1. В данном случае дифференциал фреше имеет наиболее простой вид

$$
P_{w}^{\prime}(0, \lambda) h=\left(-\Delta h-\lambda h,\left.h\right|_{\partial \Omega}\right),
$$

и подпространство $N(0, \lambda)$ определяется следующей краевой задачей

$$
\begin{cases}-\Delta h=\lambda h, & (x, y) \in \Omega, \\ h=0, & (x, y) \in \partial \Omega .\end{cases}
$$

Следовательно, в силу леммы 3.1 бифуркационными значениями $\lambda$ могут быть только собственные значения оператора $A(0) h=-\Delta h$ на подпространстве $C_{0}^{m, \alpha}(\bar{\Omega})$. Собственные значения оператора $A(0)$ на круге хорошо известны. Это $\left\{\lambda_{k j}: k=0,1,2, \ldots, j=1,2, \ldots\right\}$ - множество нулей функций Бесселя

$$
J_{k}(\lambda)=\frac{1}{\pi} \int_{0}^{\pi} \cos (\lambda \sin t-k t) d t
$$

Если $k=0$, то $\operatorname{dim} N\left(0, \lambda_{0 j}\right)=1$ для всех $j=1,2, \ldots$ и подпространство $N\left(0, \lambda_{0 j}\right)$ определяется базисным элементом

$$
e_{j}(x, y)=C_{j} J_{0}\left(\lambda_{0 j} r\right), \quad C_{j}=\left(\iint_{\Omega} J_{0}^{2}\left(\lambda_{0 j} r\right) d x d y\right)^{-1}
$$

где $r$ - полярньг̆ радиус точки $(x, y)$, см. рис. 4 а.

TEOPEMA 5.1. Пусть $\lambda=\lambda_{0 j}$, әде $j=1,2, \ldots u e_{j}(x, y)=C_{j} J_{0}\left(\lambda_{0 j} r\right)$.

Тогда $\left(0, \lambda_{0 j}\right)$ - точка бифуркачии для уравнения $P(w, \lambda)=(0,0)$, в которой от семейства решений

$$
\Gamma_{0}=\{(0, \lambda): \lambda \in \mathbb{R}\}
$$

отделяются две ненулевие ветви (см. рис. 5) $\Gamma_{1 j}^{*} u \Gamma_{2 j}^{*}$, которые пересекают ветвь $\Gamma_{0}$ под прямым углом и имеют следующее асимптотическое разложенuе

$$
\begin{gathered}
\Gamma_{i j}^{*}=\left\{\left(w_{i j}^{*}(\lambda), \lambda\right): \lambda \in\left[\lambda_{0 j}, \lambda_{0 j}+\varepsilon\right)\right\}, \\
w_{i j}^{*}(\lambda)=(-1)^{i+1} \frac{e_{j}}{\sqrt{\lambda_{j}^{*}}}\left(\lambda-\lambda_{0 j}\right)^{1 / 2}+o\left(\lambda-\lambda_{0 j}\right)^{1 / 2},
\end{gathered}
$$

где $i=1,2 u$

$$
\lambda_{j}^{*}=\frac{1}{3 !} \Phi_{\xi}^{(4)}\left(0, \lambda_{0 j}\right)=\frac{1}{2} \iint_{\Omega}\left|\nabla e_{j}(x, y)\right|^{4} d x d y>0 .
$$




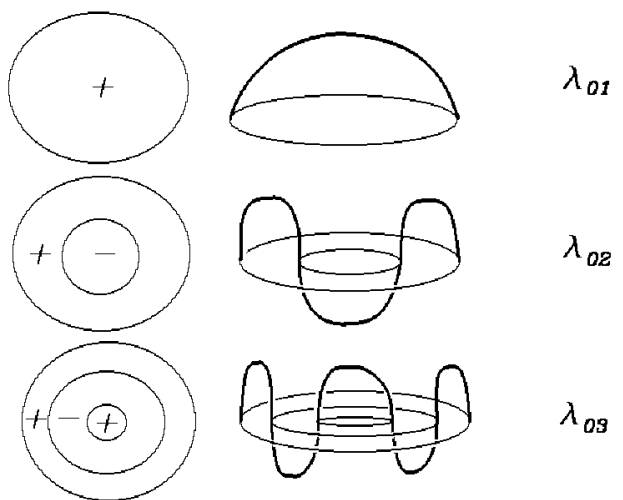

Рис. 4a

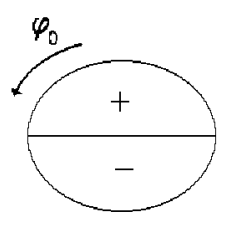

$\lambda_{11}$

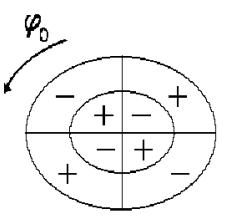

$\lambda_{22}$

Рис. $4 \mathrm{~b}$

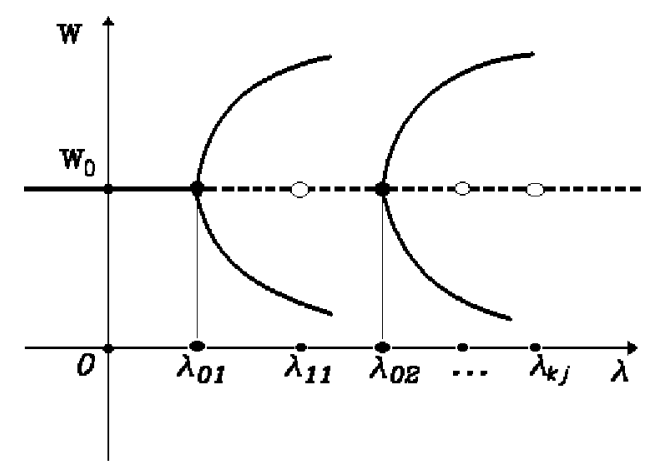

Рис. 5

СлеДСтвиЕ. В критической точке $\left(0, \lambda_{0 j}\right), j=1,2, \ldots$, ключевая функиия $\Phi\left(\xi, \lambda_{0 j}\right)$ имеет особенность типа $\mathrm{A}_{3}$ ("сборка")

$$
\begin{gathered}
\Phi\left(0, \lambda_{0 j}\right)=\pi, \quad \Phi_{\xi}^{\prime}\left(0, \lambda_{0 j}\right)=\Phi_{\xi}^{\prime \prime}\left(0, \lambda_{0 j}\right)=\Phi_{\xi}^{\prime \prime \prime}\left(0, \lambda_{0 j}\right)=0, \\
\Phi_{\xi}^{(4)}\left(0, \lambda_{0 j}\right)=3 C_{j}^{4} \iint_{\Omega}\left|\nabla J_{0}\left(\lambda_{0 j} r\right)\right|^{4} d x d y \neq 0 .
\end{gathered}
$$

Теорема 5.1 и ее следствие доказьваются непосредственной проверкой условий теорем 2.2, 3.1, 4.1 .

ЗАМЕЧАнИЕ. Если граница $\left.w\right|_{\partial \Omega}$ поверхности раздела претерпевает малъе деформачии при изменении параметра $\mu$ вблизи точки бифуркации $\left(0, \lambda_{0}, 0\right)$, то будет справедлива теорема 4.2 .

Число Милнора, коразмерность особенности, равно двум: $M=2$. Следовательно, найденная бифуркационная поверхность топологически неустойчива и будет разрушаться при возникновении дополнительных малых возмущений. Причина неустойчивости - недостаточное количество параметров. Бифуркационная поверхность $\xi^{3}-\xi \lambda+\eta=0$ для миниверсальной деформации типа $\mathrm{A}_{3}$ показана на рис. 6а. Бифуркационная поверхность типа $\xi^{3}-\xi\left(\lambda-\lambda_{0}\right)=0$, найденная по теореме Крендала-Рабиновича (см. рис. 6b), является ее однопараметрической частью. Следовательно, однопараметрическое семейство уравнений 
$P(w, \lambda)=(0,0)$ может быть включено в большее двупараметрическое семейство уравнений $Q(w, \lambda, \eta)=(0,0)$ такое, что $Q(w, \lambda, 0)=P(w, \lambda)$ и бифуркационная поверхность в пространстве $C^{m \alpha}(\bar{\Omega}) \times \mathbb{R}^{2}$ в окрестности точки $\left(0, \lambda_{0}, 0\right)$ является топологически устойчивой при возникновении дополнительных малых возмушений и подобна поверхности типа $\xi^{3}-\xi\left(\lambda-\lambda_{0}\right)+\eta=0$ в $\mathbb{R}^{3}$.

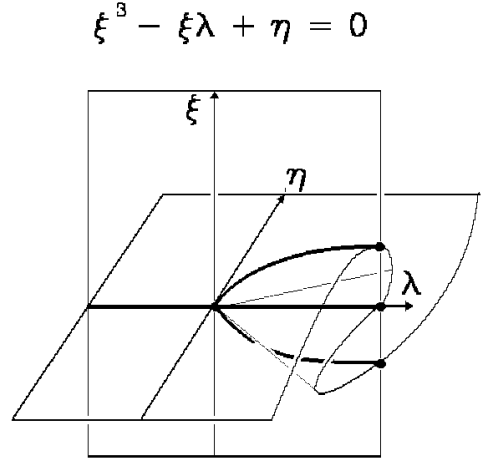

Рис. 6а

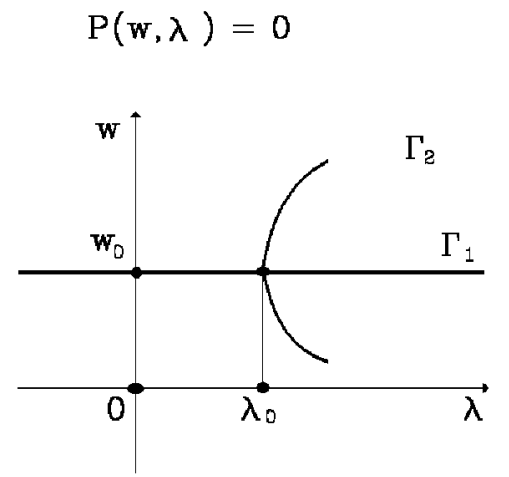

Рис. $6 \mathrm{~b}$

Если $k=1,2, \ldots$, то $\operatorname{dim} N\left(0, \lambda_{k j}\right)=2$ для всех $j=1,2, \ldots$ и подпространство $N\left(0, \lambda_{k j}\right)$ определяется базисньми элементами

$$
\begin{aligned}
& e_{1 k j}(x, y)=C_{1 k j} J_{k}\left(\lambda_{k j} r\right) \cos (k \varphi), \\
& e_{2 k j}(x, y)=C_{2 k j} J_{k}\left(\lambda_{k j} r\right) \sin (k \varphi),
\end{aligned}
$$

где $(r, \varphi)$ - полярные координаты точки $(x, y)$. С другой стороны, это же подпространство может быть представлено в виде

$$
N\left(0, \lambda_{k j}\right)=\left\{C J_{k}\left(\lambda_{k j} r\right) \sin \left(k \varphi+\varphi_{0}\right): C, \varphi_{0} \in \mathbb{R}\right\}
$$

Представление (5.8) означает, что $N\left(0, \lambda_{k j}\right)$ инвариантно относительно действия группы $\mathrm{SO}(2)$ на пространстве $\mathbb{R}^{2}(x, y)$, см. рис. $4 \mathrm{~b}$. Если функция $w(x, y) \in$ $N\left(0, \lambda_{k j}\right)$, тогда $w(g(x, y)) \in N\left(0, \lambda_{k j}\right)$ для всех матриц $G \in \mathrm{SO}(2)$, где

$$
g(x, y)=\left(G \times\left[\begin{array}{l}
x \\
y
\end{array}\right]\right)^{\top}
$$

Вопрос о бифуркации в этом случае остается открытым.

Исследуем случай, когда область $\Omega$ - квадрат (см. рис. 7а)

$$
\Omega=\{(x, y):-1<x<1,-1<y<1\}
$$

Подпространство $N(0, \lambda)$ в $W_{p}^{2}(\Omega), p>2$, определяется краевой задачей $(5.2)$. Следовательно, в силу леммы 3.1 бифуркационными значениями $\lambda$ могут быть 


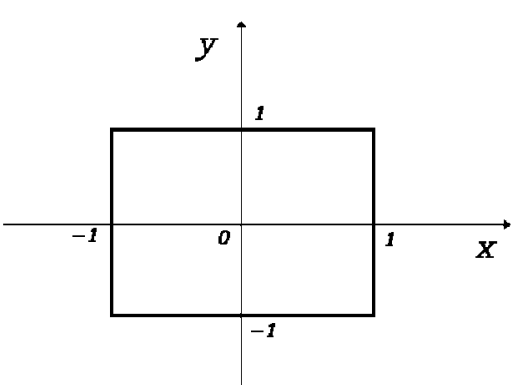

Рис. $7 \mathrm{a}$

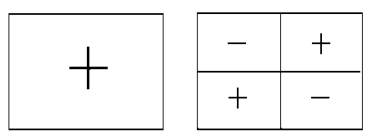

$\lambda_{11}$

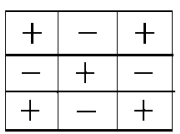

$\lambda_{33}$
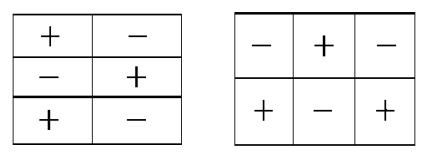

$\lambda_{32}=\lambda_{23}$

Рис. $7 \mathrm{~b}$

только собственные значения оператора $A(0) h=-\Delta h$ на подпространстве $W_{p 0}^{2}(\Omega)$ функций с нулевыми следами на границе.

Собственные значения оператора $A(0)$ на квадрате хорошо известны. Это

$$
\lambda_{k m}=\left(\frac{\pi}{2} k\right)^{2}+\left(\frac{\pi}{2} m\right)^{2}, \quad k, m \in \mathbb{N}
$$

и $\operatorname{dim} N\left(0, \lambda_{k}, m\right)$ равна числу различных пар натуральных чисел $(p, q)$ таких, что $p^{2}+q^{2}=k^{2}+m^{2}$. Например, $\operatorname{dim} N\left(0, \lambda_{1,7}\right)=3$ в силу $1^{2}+7^{2}=7^{2}+1^{2}=$ $5^{2}+5^{2}=50$.

Если $k=m$ и $\operatorname{dim} N\left(0, \lambda_{k k}\right)=1$, то подпространство $N\left(0, \lambda_{k k}\right)$ определяется базисным элементом

$$
e_{k}(x, y)=C_{k} v_{k}(x) v_{k}(y),
$$

см. рис. $7 \mathrm{~b}$, где $\left\|e_{k}\right\|=1$ в пространстве $L_{2}(\Omega)$ и

$$
v_{k}(t)=\left\{\begin{array}{l}
\cos \left(k \frac{\pi}{2} t\right), \text { если } k \text { нечетно, } \\
\sin \left(k \frac{\pi}{2} t\right), \text { если } k \text { четно. }
\end{array}\right.
$$

В случае однократного вырождения применимы теоремы $3.1,3.2,4.1$. Получаем следуюшие теоремы о бифуркациях.

TеOPEMA 5.2. Пусть $\lambda=\lambda_{k k} u \operatorname{dim} N\left(0, \lambda_{k k}\right)=1, c \mathcal{M}$. (5.10)-(5.11).

Тогда $\left(0, \lambda_{k k}\right)$ - точка бифуркачии для уравнения $P(w, \lambda)=(0,0)$, в которой от семейства решений

$$
\Gamma_{0}=\{(0, \lambda): \lambda \in \mathbb{R}\}
$$

отделяются две ненулевые ветви (см. рис. 8) $\Gamma_{1 k}^{*} u \Gamma_{2 k}^{*}$, которые пересекают ветвь $\Gamma_{0}$ под прямым углом и имеют следующее асимптотическое разложение

$$
\begin{gathered}
\Gamma_{i k}^{*}=\left\{\left(w_{i k}^{*}(\lambda), \lambda\right): \lambda \in\left[\lambda_{0 k}, \lambda_{0 k}+\varepsilon\right)\right\}, \quad i=1,2, \\
w_{1 k}^{*}(\lambda)=+\frac{e_{k}}{\sqrt{\lambda_{k}^{*}}}\left(\lambda-\lambda_{k k}\right)^{1 / 2}+o\left(\lambda-\lambda_{k k}\right)^{1 / 2}, \\
w_{2 k}^{*}(\lambda)=-\frac{e_{k}}{\sqrt{\lambda_{k}^{*}}}\left(\lambda-\lambda_{k k}\right)^{1 / 2}+o\left(\lambda-\lambda_{k k}\right)^{1 / 2}
\end{gathered}
$$


$2 \partial e$

$$
\lambda_{k}^{*}=\frac{1}{3 !} \Phi_{\xi}^{(4)}\left(0, \lambda_{k k}\right)=\frac{1}{2} \iint_{\Omega}\left|\nabla e_{k}(x, y)\right|^{4} d x d y=\frac{3}{64} k \pi .
$$

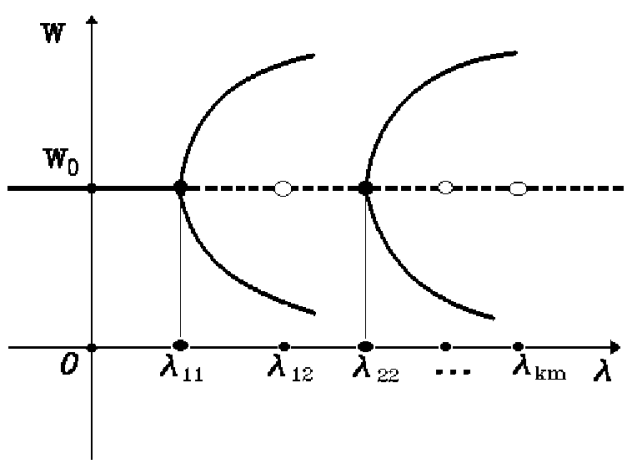

Рис. 8

СлЕДСТВИЕ. Пусть $\operatorname{dim} N\left(0, \lambda_{k k}\right)=1$. Тогда, в критической точке $\xi_{0}=0$ ключевая функиия $\Phi\left(\xi, \lambda_{k k}\right)$ имеет особенность типа $\mathrm{A}_{3}$ ("сборка")

$$
\begin{gathered}
\Phi\left(0, \lambda_{k k}\right)=\pi, \quad \Phi_{\xi}^{\prime}\left(0, \lambda_{k k}\right)=0, \quad \Phi_{\xi}^{\prime \prime}\left(0, \lambda_{k k}\right)=0 \\
\Phi_{\xi}^{\prime \prime \prime}\left(0, \lambda_{k k}\right)=0, \quad \Phi_{\xi}^{(4)}\left(0, \lambda_{k k}\right)=\frac{9}{32} k \pi
\end{gathered}
$$

ЗАмЕчАниЕ. Если граница $\left.w\right|_{\partial \Omega}$ поверхности раздела претерпевает малые деформации при изменении параметра $\mu$ вблизи точки бифуркации $\left(0, \lambda_{0}, 0\right)$, то будет справедлива теорема 4.2.

Из непосредственного исследования формулы (2.5) для второй вариации $\delta^{2} E$ вытекает, что тривиальное решение $w_{0}(x, y) \equiv 0$ теряет свою устойчивость после первой точки бифуркации $\lambda_{11}$, в которой от него ответвляются два семейства устойчивых решений (см. рис. 8).

Однако, в остальных однократных точках бифуркации $\lambda_{k k}, k=2,3, \ldots$, от $w_{0}(x, y) \equiv 0$ тоже могут ответвляться устойчивые решения $(5.11)$, имеющие $k$ продольных и $k$ поперечных волн (см. рис. $7 \mathrm{~b})$. В физических экспериментах такие поверхности раздела могут возникнуть, если параметр $\lambda$ изменился достаточно быстро (скачком).

Если $k \neq m$ и $\operatorname{dim} N\left(0, \lambda_{k m}\right)=2$, то подпространство $N\left(0, \lambda_{k m}\right)$ определяется базисными элементами (см. рис. $7 \mathrm{~b})$

$$
\begin{aligned}
& e_{1}(x, y)=v_{k}(x) v_{m}(y), \\
& e_{2}(x, y)=v_{m}(x) v_{k}(y) .
\end{aligned}
$$

Заметим, что в этом случае подпространство $N\left(0, \lambda_{k m}\right)$ инвариантно относительно действия инволюции $x$ и $y$. Если функция $w(x, y) \in N\left(0, \lambda_{k m}\right)$, тогда $w(y, x) \in N\left(0, \lambda_{k m}\right)$.

Вопрос о бифуркации в этом случае остается открытым. 


\section{Список литературы}

1. Дао Чонг Тхи, Фоменко А. Т. Минимальные поверхности, стратифицированые многообразия и проблема Плато. М.: Наука, 1987.

2. Фоменко А. Т. Вариационные проблемы в топологии. М.: Изд-во МГУ, 1984.

3. Dissan V. E. B. On the spreading of liquids on solid surfaces: Static and dynamic contact lines // Ann. Rev. Fluid Mech. 1979. V. 11. P. 371-400.

4. Dissan V.E.B., Davis S. H. Stability in systems with moving contact lines // J. Fluid Mech. 1986. V. 173. P. 115-130.

5. Finn R. Equilibrium capillary surfaces: Springer-Verlag, 1986.

6. Бабский В.Г., Копачевский Н.Д., Мышкис А.Д., Слобожанин Л.А., Таупсов А. Д. Гидромеханика невесомости. М.: Наука, 1975.

7. Babskii V. G., Kopachevskii N.D., Myshkis A.D., Slobozhanin L.A., Tyuptsov A.D. On some unsolved problems of zero dimensional hydromechanics // Nonlinear Anal. 1980. V. 4. P. 607-621.

8. Румянцев B. B., Владимиров В. А. Теорема Лагранжа для твердого тела с полостью, заполненной идеальной жидкостью // Прикладная математика и механика. 1989. Т. 53. № 4 . C. $608-612$.

9. Румянцев B. В., Владимиров В. А. Теорема Лагранжа для твердого тела с полостью, заполненной вязкой жидкостью // Прикладная математика и механика. 1990. Т. 54. № 2. C. $190-200$.

10. Poston T. A global theorem for nonlinear eigenvalue problems and applications // Contrib. Nonlinear Fcl. Anal.: Academic Press, 1971. P. 11-36.

11. Beeson M.J., Tromba A.J. The cusp catastrophe of Thom in the bifurcation on minimal surfaces // Manuscripta Math. 1984. V. 46. № 1-3. P. 273-308.

12. Фоменко A.T., Тужилин A. А. Многозначные отображения, минимальные поверхности и мыльные пленки // Вестн. МГУ. Сер. I, матем., мех. 1986. Т. 3. С. 3-12.

13. Тужилин A. A. О бифуркации двумерных минимальных поверхностей при двупараметрической вариации контура // Геометрия, дифференциальные уравнения, механика. М.: Изд-во МГУ, 1986. Р. 140-145.

14. Buch J. Bifurcation von Minimalflachen und elementare katastrophen // Manuscripta Math. 1986. V. 55. № 3-4.

15. Borisovich A. Yu. Functional-Topological Properties of the Plateau Operator and Applications to the Study of Bifurcations in Problems of Geometry and Hydrodynamics // Adv. Soviet Math. 1993. V. 15. P. 287-330.

16. Бернштейн C. Н. Дифференциальные уравнения с частными производными. М.: Изд-во AH CCCP, 1960.

17. Борисович А.Ю., Звягин В.Г. О глобальной обратимости нелинейных операторов, порождаемых краевыми задачами // Приближенные методы исследования дифференциальных уравнений и их приложения. Куйбышев: КГУ, 1983. Р. 27-33.

18. Gilbarg D., Trudinger N.S. Elliptic Partial Differential Equations of Second Order: Springer-Verlag, 1983.

19. Кондратьев B. A. Краевые задачи для эллиптических уравнений в областях с коническими и угловыми точками // Труды ММО. 1967. Т. 16. С. 209-293.

20. Crandall M.G., Rabinowitz P.H. Bifurcation from simple eigenvalues // Funct. Anal. 1971. V. 8. P. 321-340.

21. Nirenberg L. Topics in nonlinear functional analysis. New York: Courant Inst. of Math. Sci., 1974.

22. Красносельский М.A. Топологические методы в теории нелинейных интегралњњых уравнений. М.: Гостехиздат, 1956.

23. Rabinovich P. H. The Problem of Plateau, an introduction to the whole Mathematics. Mimeographical Notes. Summer conference, Trieste, 1972.

24. Ize I. A. Bifurcation theory for Fredholm operators // Mem. Amer. Math. Soc. 1976. V. 174 . 
25. Арнольд В.И., Гусейн-Заде С. М., Варченко А.Н. Особенности дифференцируемых отображений. Т. I, II. М.: Наука, 1982.

26. Sapronov Yu. I. Branching of solutions of smooth Fredholm equations // Lecture Notes in Math. 1982. V. 1108. P. 31-54.

27. Вайнберг M. M., Треногин B. A. Теория ветвления решений нелинейных уравнений. М.: Наука, 1969.

г. Воронеж

Поступила в редакцию 28.05.1996 